\title{
Stabilization of the Brylinski-Kostant filtration and limit of Lusztig $q$-analogues
}

\author{
Cédric Lecouvey
}

Received: 17 April 2007 / Accepted: 9 August 2007 / Published online: 18 September 2007

(C) Springer Science+Business Media, LLC 2007

\begin{abstract}
Let $G$ be a simple complex classical Lie group with Lie algebra $\mathfrak{g}$ of rank $n$. We show that the coefficient of degree $k$ in the Lusztig $q$-analogue $K_{\lambda, \mu}^{\mathfrak{g}}(q)$ associated to the fixed partitions $\lambda$ and $\mu$ stabilizes for $n$ sufficiently large. As a consequence, we obtain the stabilization of the dimensions in the Brylinski-Kostant filtration associated to any dominant weight. We then introduce, for each pair of partitions $(\lambda, \mu)$, formal series which can be regarded as natural limits of the Lusztig $q$-analogues. We give a duality property for these limits and recurrence formulas which permit notably to derive explicit expressions when $\lambda$ is a row or a column partition.
\end{abstract}

Keywords Brylinski filtration $\cdot q$-analogues $\cdot$ Lusztig polynomials $\cdot q$-series

\section{Introduction}

The multiplicity $K_{\lambda, \mu}$ of the weight $\mu$ in the irreducible finite-dimensional representation $V^{\mathfrak{g}}(\lambda)$ of the simple Lie group $G$ with Lie algebra $\mathfrak{g}$ can be written in terms of the ordinary Kostant partition function $\mathcal{P}$ defined by the equality

$$
\prod_{\alpha \text { positive root }} \frac{1}{\left(1-e^{\alpha}\right)}=\sum_{\beta} \mathcal{P}(\beta) e^{\beta}
$$

where $\beta$ runs on the set of nonnegative integral combinations of positive roots of $\mathfrak{g}$. Thus $\mathcal{P}(\beta)$ is the number of ways the weight $\beta$ can be expressed as a sum of positive roots. Then, from the Weyl character formula, one derives the identity

$$
K_{\lambda, \mu}^{\mathfrak{g}}=\sum_{w \in W^{\mathfrak{g}}}(-1)^{\ell(w)} \mathcal{P}(w(\lambda+\rho)-(\mu+\rho))
$$

C. Lecouvey $(\bowtie)$

Laboratoire de Mathématiques Pures et Appliquées Joseph Liouville, B.P. 699, 62228 Calais Cedex, France

e-mail: Cedric.Lecouvey@1mpa.univ-littoral.fr 
where $W^{\mathfrak{g}}$ is the Weyl group of $\mathfrak{g}$.

The Lusztig $q$-analogue of weight multiplicity $K_{\lambda, \mu}^{\mathfrak{g}}(q)$ is obtained by substituting the ordinary Kostant partition function $\mathcal{P}$ by its $q$-analogue $\mathcal{P}_{q}^{\mathfrak{g}}$ in (1). Namely $\mathcal{P}_{q}^{\mathfrak{g}}$ is defined by the equality

$$
\prod_{\alpha \text { positive root }} \frac{1}{\left(1-q e^{\alpha}\right)}=\sum_{\beta} \mathcal{P}_{q}^{\mathfrak{g}}(\beta) e^{\beta}
$$

and we have

$$
K_{\lambda, \mu}^{\mathfrak{g}}(q)=\sum_{w \in W^{\mathfrak{g}}}(-1)^{\ell(w)} \mathcal{P}_{q}^{\mathfrak{g}}(w(\lambda+\rho)-(\mu+\rho))
$$

As shown by Lusztig [14], $K_{\lambda, \mu}^{\mathfrak{g}}(q)$ is a polynomial in $q$ with nonnegative integer coefficients. Many interpretations of the Lusztig $q$-analogues exist. In particular, they can be obtained from the Brylinski-Kostant filtration of weight spaces [1]. The polynomials $K_{\lambda, \emptyset}^{\mathfrak{g}}(q)$ appear in the graded character of the harmonic polynomials associated to $\mathfrak{g}[5]$. We also recover the Lusztig $q$-analogues as the coefficients of the expansion of the Hall-Littlewood polynomials on the basis of Weyl characters [7]. This notably permits to prove that they are affine Kazhdan-Lusztig polynomials. In [11] Lascoux and Schützenberger have obtained a combinatorial expression for $K_{\lambda, \mu}^{\mathfrak{g l} l_{n}}(q)$ in terms of the charge statistic on the semistandard tableaux of shape $\lambda$ and evaluation $\mu$. By using the combinatorics of crystal graphs introduced by Kashiwara and Nakashima [6], we have also established similar formulas [12, 13] for the Lusztig $q$-analogues associated to the symplectic and orthogonal Lie algebras when $(\lambda, \mu)$ satisfies restrictive constraints.

Consider $\lambda, \mu$ two partitions of length at most $m$. These partitions can be regarded as dominant weights for $\mathfrak{g}=\mathfrak{g l}_{n}, \mathfrak{s o}_{2 n+1}, \mathfrak{s p}_{2 n}$ or $\mathfrak{s o}_{2 n}$ when $n \geq m$. Then, $K_{\lambda, \mu}^{\mathfrak{g l}_{n}}(q)$ does not depend on the rank $n$ considered. Such a property does not hold for the Lusztig $q$-analogues $K_{\lambda, \mu}^{\mathfrak{g}}(q)$ when $\mathfrak{g}=\mathfrak{s o}_{2 n+1}, \mathfrak{s p}_{2 n}$ or $\mathfrak{s o}_{2 n}$ which depend in general on the rank of the Lie algebra considered. Write

$$
K_{\lambda, \mu}^{\mathfrak{g}}(q)=\sum_{k \geq 0} K_{\lambda, \mu}^{\mathfrak{g}, k} q^{k}
$$

We first establish in this paper that for $\mathfrak{g}=\mathfrak{s o}_{2 n+1}, \mathfrak{s p}_{2 n}$ or $\mathfrak{s o}_{2 n}$, the coefficient $K_{\lambda, \mu}^{\mathfrak{g}, k}$ stabilizes when $n$ tends to infinity. More precisely, $K_{\lambda, \mu}^{\mathfrak{g}, k}$ does not depend on the rank $n$ of $\mathfrak{g}$ providing $n \geq 2 k+a$ where $a$ is the number of nonzero parts of $\mu$ (Theorem 4.3.1). Note that this result cannot be obtained by simply taking the limit when $n$ tends to infinity in (2). Indeed, the number of decompositions of a given weight as a sum of $k$ positive roots may strictly increase with $n$. Hence, there is no analogue of the Kostant partition function in infinite rank (but see Remark (i) in Section 6.2). By Brylinski's interpretation of the coefficients $K_{\lambda, \mu}^{\mathfrak{g}, k}$ [1], one then obtains that the dimension of the $k$-th component of the Brylinski-Kostant filtration associated to the finite-dimensional irreducible representations of $\mathfrak{g}=\mathfrak{s o}_{2 n+1}, \mathfrak{s p}_{2 n}$ or $\mathfrak{s o}_{2 n}$ stabilizes for $n$ sufficiently large (Theorem 4.4.2). Observe that this stabilization is immediate 
for $\mathfrak{g}=\mathfrak{g l}_{n}$ since the polynomials $K_{\lambda, \mu}^{\mathfrak{g l}_{n}}(q)$ do not depend on $n$. For $\mathfrak{g}=\mathfrak{s o}_{2 n+1}, \mathfrak{s p}_{2 n}$ or $\mathfrak{s o}_{2 n}$ the Brylinski-Kostant filtration depends in general on the rank considered and it seems difficult to obtain the dimension of its components by direct computations. Our method is as follows. We obtain the explicit decomposition of the symmetric algebra $S(\mathfrak{g})$ considered as a $G$-module into its irreducible components by using identities due to Littlewood. This permits to show that the multiplicities appearing in the decomposition of the $k$-th graded component $S^{k}(\mathfrak{g})$ of $S(\mathfrak{g})$ do not depend on the rank $n$ of $\mathfrak{g}$ providing $n$ is sufficiently large. Observe that this stabilization also follows from a more general result due to Hanlon [3]. Nevertheless our computations, based on Littlewood's identities, have the interest to yield simple explicit formulas in terms of the Littlewood-Richardson coefficients for the decomposition of $S^{k}(\mathfrak{g})$ in large rank. Thanks to a classical result by Kostant, we establish a similar result for the $k$-th graded component $H^{k}(\mathfrak{g})$ of the space $H(\mathfrak{g})$ of $G$-harmonic polynomials. These stabilization properties are equivalent to the existence of a limit in infinitely many variables for the graded characters associated to $S(\mathfrak{g})$ and $H(\mathfrak{g})$. The limits so obtained are formal series with coefficients in the ring of universal characters introduced by Koike and Terada. From Hesselink expression [5] of the graded character of $H(\mathfrak{g})$, one then derives that $K_{\lambda, \emptyset}^{\mathfrak{g}, k}$ stabilizes for $n$ sufficiently large. By using Morris-type recurrence formulas for the Lusztig $q$-analogues [13], we prove that this is also true for the coefficients $K_{\lambda, \mu}^{\mathfrak{g}, k}$ where $\mu$ is a fixed nonempty partition. We also observe that these formulas permit to give an explicit lower bound for the degree of the $q$-analogues $K_{\lambda, \mu}^{\mathfrak{g}}(q)$ such that $K_{\lambda, \mu}^{\mathfrak{g}}(q) \neq 0$. We establish that the limits of the coefficients $K_{\lambda, \mu}^{\mathfrak{s o}_{2 n+1}, k}$ and $K_{\lambda, \mu}^{\mathfrak{s o}_{2 n}, k}$ are the same. Write $K_{\lambda, \mu}^{\mathfrak{s o}, k}$ and $K_{\lambda, \mu}^{\mathfrak{s p}, k}$ respectively for the limits of the coefficients $K_{\lambda, \mu}^{\mathfrak{s o}_{2 n+1}, k}$ and $K_{\lambda, \mu}^{\mathfrak{s p}_{2 n}, k}$ when $n$ tends to infinity.

The stabilization property of the coefficients $K_{\lambda, \mu}^{\mathfrak{g}, k}$ suggests then to introduce the formal series

$$
K_{\lambda, \mu}^{\mathfrak{s o}}(q)=\sum_{k \geq 0} K_{\lambda, \mu}^{\mathfrak{s o}, k} q^{k} \quad \text { and } \quad K_{\lambda, \mu}^{\mathfrak{s p}}(q)=\sum_{k \geq 0} K_{\lambda, \mu}^{\mathfrak{s p}, k} q^{k}
$$

These series belong to $\mathbb{N}[[q]]$ and can be regarded as natural limits of the polynomials $K_{\lambda, \mu}^{\mathfrak{g}}(q)$. As far as the author is aware, the first occurrence of such limits for the Lusztig $q$-analogues was in [3] in the particular case $\mu=\emptyset$. We establish a duality between the formal series $K_{\lambda, \emptyset}^{\mathfrak{s o}}(q)$ and $K_{\lambda, \emptyset}^{\mathfrak{s p}}(q)$ (Theorem 5.3.1). Namely, we have

$$
K_{\lambda, \emptyset}^{\mathfrak{s o}}(q)=K_{\lambda^{\prime}, \emptyset}^{\mathfrak{s p}}(q)
$$

where $\lambda^{\prime}$ is the conjugate partition of $\lambda$. Note that (3) do not hold in general if we replace the formal series $K_{\lambda, \mu}^{\mathfrak{s o}}(q)$ and $K_{\lambda, \mu}^{\mathfrak{s p}}(q)$ by the polynomials $K_{\lambda, \mu}^{\mathfrak{g}, k}(q)$. We also give recurrence formulas (39), (40) for the series $K_{\lambda, \mu}^{\mathfrak{s o}}(q)$ and $K_{\lambda, \mu}^{\mathfrak{s p}}(q)$ which permit efficient recursive computations. Thanks to these recurrence formulas, one derives simple expressions for the formal series $K_{\lambda, \mu}^{\mathfrak{s o}}(q)$ and $K_{\lambda, \mu}^{\mathfrak{s p}}(q)$ when $\lambda$ is a single row or a single column partition (Proposition 5.4.1). Such simple formulas seem not to exist for the Lusztig $q$-analogues $K_{\lambda, \mu}^{\mathfrak{g}}(q)$ even in the cases when $\lambda$ is a single column or a single row partition. Moreover the duality (3) is false in general for the 
polynomials $K_{\lambda, \mu}^{\mathfrak{g}}(q)$. This suggests that the study of the series $K_{\lambda, \mu}^{\mathfrak{s o}}(q)$ and $K_{\lambda, \mu}^{\mathfrak{s p}}(q)$ which is initiated in this paper, could be easier than that of the Lusztig $q$-analogues.

In this paper we restrict ourselves to the polynomial representations of $S O_{N}(\mathbb{C})$ and $S p_{N}(\mathbb{C})$. Nevertheless, our results can also be extended to the $q$-analogues of weight multiplicities corresponding to the irreducible finite-dimensional rational representations of $G L_{n}(\mathbb{C})$. Limits of such $q$-analogues in large rank for the zero weight have been previously studied, notably by Gupta [2] and Hanlon [3]. They can be obtained from the Cauchy formula which then plays the role of the Littlewood identities used in Section 3. The existence of such limits for any dominant weight is then deduced from the Morris recurrence formula for the Lusztig $q$-analogues associated to the root systems of type $A$ by using arguments similar to those of Sections 4 and 5 . This also yields the stabilization of the dimensions in the Brylinski-Kostant filtration and recurrence formulas for the limits obtained.

The paper is organized as follows. In Section 2 we recall the necessary background on symplectic and orthogonal Lie algebras, universal characters, and Lusztig $q$-analogues which is needed in the sequel. In Section 3 we introduce universal graded characters as limits in infinitely many variables for the graded characters associated to $S(\mathfrak{g})$ and $H(\mathfrak{g})$. We obtain the stabilization property of the coefficients $K_{\lambda, \mu}^{\mathfrak{g}, k}$ in Section 4 and reformulate this result in terms of the Brylinski-Kostant filtration. In Section 5, we introduce the formal series $K_{\lambda, \mu}^{\mathfrak{s o}}(q)$ and $K_{\lambda, \mu}^{\mathfrak{s p}}(q)$, establish recurrence formulas which permit to compute them by induction, prove the duality (3) and give explicit formulas for $K_{\lambda, \mu}^{\mathfrak{s o}}(q)$ and $K_{\lambda, \mu}^{\mathfrak{s p}}(q)$ when $\lambda$ is a row or a column partition.

\section{Background}

\subsection{Convention for the root systems of types $B, C$ and $D$}

In the sequel $G$ is one of the complex Lie groups $S p_{2 n}, S O_{2 n+1}$ or $S O_{2 n}$ and $\mathfrak{g}$ is its Lie algebra. We follow the convention of [9] to realize $G$ as a subgroup of $G L_{N}$ and $\mathfrak{g}$ as a subalgebra of $\mathfrak{g l}_{N}$ where

$$
N= \begin{cases}2 n & \text { when } G=S p_{2 n}, \\ 2 n+1 & \text { when } G=S O_{2 n+1}, \\ 2 n & \text { when } G=S O_{2 n} .\end{cases}
$$

With this convention the maximal torus $T$ of $G$ and the Cartan subalgebra $\mathfrak{h}$ of $\mathfrak{g}$ coincide respectively with the subgroup and the subalgebra of diagonal matrices of $G$ and $\mathfrak{g}$. Similarly the Borel subgroup $B$ of $G$ and the Borel subalgebra $\mathfrak{b}_{+}$of $\mathfrak{g}$ coincide respectively with the subgroup and subalgebra of upper triangular matrices of $G$ and $\mathfrak{g}$. This gives the triangular decomposition $\mathfrak{g}=\mathfrak{b}_{+} \oplus \mathfrak{h} \oplus \mathfrak{b}_{-}$for the Lie algebra $\mathfrak{g}$. Let $e_{i}, h_{i}, f_{i}, i \in\{1, \ldots, n\}$ be a set of Chevalley generators such that $e_{i} \in \mathfrak{b}_{+}, h_{i} \in \mathfrak{h}$ and $f_{i} \in \mathfrak{b}_{-}$for any $i$.

Let $d_{N}$ be the linear subspace of $\mathfrak{g l}_{N}$ consisting of the diagonal matrices. For any $i \in\{1, \ldots, n\}$, write $\varepsilon_{i}$ for the linear map $\varepsilon_{i}: d_{N} \rightarrow \mathbb{C}$ such that $\varepsilon_{i}(D)=\delta_{i}$ for any diagonal matrix $D$ whose $(i, i)$-coefficient is $\delta_{i}$. Then $\left(\varepsilon_{1}, \ldots, \varepsilon_{n}\right)$ is an orthonormal 
basis of the Euclidean space $\mathfrak{h}_{\mathbb{R}}^{*}$ (the real part of $\mathfrak{h}^{*}$ ). We denote by $<\cdot, \cdot>$ the usual scalar product on $\mathfrak{h}_{\mathbb{R}}^{*}$. For any $\beta \in \mathfrak{h}_{\mathbb{R}}^{*}$, we write $\beta=\left(\beta_{1}, \ldots, \beta_{n}\right)$ for the coordinates of $\beta$ on the basis $\left(\varepsilon_{1}, \ldots, \varepsilon_{n}\right)$.

Let $R$ be the root system associated to $G$. We can take for the simple roots of $\mathfrak{g}$

$$
\left\{\begin{array}{l}
\Sigma^{+}=\left\{\alpha_{n}=\varepsilon_{n} \text { and } \alpha_{i}=\varepsilon_{i}-\varepsilon_{i+1}, i=1, \ldots, n-1\right\} \\
\quad \text { for the root system } B_{n} \\
\Sigma^{+}=\left\{\alpha_{n}=2 \varepsilon_{n} \text { and } \alpha_{i}=\varepsilon_{i}-\varepsilon_{i+1}, i=1, \ldots, n-1\right\} \\
\quad \text { for the root system } C_{n}, \\
\Sigma^{+}=\left\{\alpha_{n}=\varepsilon_{n}+\varepsilon_{n-1} \text { and } \alpha_{i}=\varepsilon_{i}-\varepsilon_{i+1}, i=1, \ldots, n-1\right\} \\
\quad \text { for the root system } D_{n} .
\end{array}\right.
$$

Then the sets of positive roots are

$$
\left\{\begin{array}{l}
R^{+}=\left\{\varepsilon_{i}-\varepsilon_{j}, \varepsilon_{i}+\varepsilon_{j} \text { with } 1 \leq i<j \leq n\right\} \cup\left\{\varepsilon_{i} \text { with } 1 \leq i \leq n\right\} \\
\quad \text { for the root system } B_{n}, \\
R^{+}=\left\{\varepsilon_{i}-\varepsilon_{j}, \varepsilon_{i}+\varepsilon_{j} \text { with } 1 \leq i<j \leq n\right\} \cup\left\{2 \varepsilon_{i} \text { with } 1 \leq i \leq n\right\} \\
\quad \text { for the root system } C_{n}, \\
R^{+}=\left\{\varepsilon_{i}-\varepsilon_{j}, \varepsilon_{i}+\varepsilon_{j} \text { with } 1 \leq i<j \leq n\right\} \\
\quad \text { for the root system } D_{n} .
\end{array}\right.
$$

We denote by $R$ the set of roots of $G$. For any $\alpha \in R$, let $\alpha^{\vee}=\frac{\alpha}{<\alpha, \alpha>}$ be the coroot corresponding to $\alpha$. The Weyl group of the Lie group $G$ is the subgroup of the permutation group of the set $\{\bar{n}, \ldots, \overline{2}, \overline{1}, 1,2, \ldots, n\}$ generated by the permutations

$$
\left\{\begin{array}{l}
s_{i}=(i, i+1)(\bar{i}, \overline{i+1}), i=1, \ldots, n-1 \text { and } s_{n}=(n, \bar{n}) \\
\quad \text { for the root systems } B_{n} \text { and } C_{n}, \\
s_{i}=(i, i+1)(\bar{i}, \overline{i+1}), i=1, \ldots, n-1 \text { and } s_{n}^{\prime}=(n, \overline{n-1})(n-1, \bar{n}) \\
\quad \text { for the root system } D_{n}
\end{array}\right.
$$

where for $a \neq b(a, b)$ is the simple transposition which switches $a$ and $b$. We identify the subgroup of $W^{\mathfrak{g}}$ generated by $s_{i}=(i, i+1)(\bar{i}, \overline{i+1}), i=1, \ldots, n-1$ with the symmetric group $S_{n}$. We denote by $\ell$ the length function corresponding to the above set of generators. The action of $w \in W^{\mathfrak{g}}$ on $\beta=\left(\beta_{1}, \ldots, \beta_{n}\right) \in \mathfrak{h}_{\mathbb{R}}^{*}$ is defined by

$$
w \cdot\left(\beta_{1}, \ldots, \beta_{n}\right)=\left(\beta_{1}^{w^{-1}}, \ldots, \beta_{n}^{w^{-1}}\right)
$$

where $\beta_{i}^{w}=\beta_{w(i)}$ if $w(i) \in\{1, \ldots, n\}$ and $\beta_{i}^{w}=-\beta_{w(\bar{i})}$ otherwise. We denote by $\rho$ the half sum of the positive roots of $R^{+}$. The dot action of $W^{\mathfrak{g}}$ on $\beta=\left(\beta_{1}, \ldots, \beta_{n}\right) \in$ $\mathfrak{h}_{\mathbb{R}}^{*}$ is defined by

$$
w \circ \beta=w \cdot(\beta+\rho)-\rho .
$$

Write $P$ and $P^{+}$for the weight lattice and the cone of dominant weights of $G$. As usual we consider the order on $P$ defined by $\beta \leq \gamma$ if and only if $\gamma-\beta \in Q^{+}$.

For any positive integer $m$, denote by $\mathcal{P}_{m}$ the set of partitions with at most $m$ nonzero parts. Let $\mathcal{P}_{m}(k), k \in \mathbb{N}$ be the subset of $\mathcal{P}_{m}$ consisting of the partitions $\lambda$ such that $|\lambda|=\lambda_{1}+\cdots+\lambda_{m}=k$. Set $\mathcal{P}=\cup_{m \in \mathbb{N}} \mathcal{P}_{m}$ and $\mathcal{P}_{m}[k]=\cup_{a \leq k} \mathcal{P}_{m}(a)$. 
Each partition $\lambda=\left(\lambda_{1}, \ldots, \lambda_{n}\right) \in \mathcal{P}_{n}$ can be identified with the dominant weight $\sum_{i=1}^{n} \lambda_{i} \varepsilon_{i}$. Then the irreducible finite-dimensional polynomial representations of $S O_{2 n+1}$ and $S p_{2 n}$ are parametrized by the partitions of $\mathcal{P}_{n}$. The irreducible finitedimensional polynomial representations of $\mathrm{SO}_{2 n}$ are parametrized by the dominant weights of $\mathcal{P}_{n} \cup \overline{\mathcal{P}}_{n}$ where

$$
\overline{\mathcal{P}}_{n}=\left\{\left(\lambda_{1}, \ldots, \lambda_{n-1},-\lambda_{n}\right) \in \mathbb{Z}^{n} \mid\left(\lambda_{1}, \ldots, \lambda_{n-1}, \lambda_{n}\right) \in \mathcal{P}_{n}\right\} .
$$

For any $\lambda \in \mathcal{P}_{n}$, we denote by $V^{\mathfrak{g}}(\lambda)$

- the irreducible finite-dimensional representation of $G$ corresponding to $\lambda$ when $\mathfrak{g}=\mathfrak{s o}_{2 n+1}, \mathfrak{s p}_{2 n}$ or when $\mathfrak{g}=\mathfrak{s o}_{2 n}$ and $\lambda_{n}=0$,

- the direct sum of the representations of $S O_{2 n}$ corresponding to the dominant weights $\lambda$ and $\bar{\lambda}=\left(\lambda_{1}, \ldots, \lambda_{n-1},-\lambda_{n}\right)$ when $\mathfrak{g}=\mathfrak{s o}_{2 n}$ and $\lambda_{n} \neq 0$.

The representation $V^{\mathfrak{g}}(1)$ associated to the partition $\lambda=(1)$ is called the vector representation of $G$. For any weight $\beta \in P$ and any partition $\lambda \in \mathcal{P}_{n}$, we write $V^{\mathfrak{g}}(\lambda)_{\beta}$ for the weight space associated to $\beta$ in $V^{\mathfrak{g}}(\lambda)$.

We denote by $Q$ the root lattice of $\mathfrak{g}$ and write $Q^{+}$for the elements of $Q$ which are linear combination of positive roots with nonnegative coefficients.

The exponents $\left\{m_{1}, \ldots, m_{n}\right\}$ of the root system $R$ verifies $m_{i}=2 i-1, i=$ $1, \ldots, n$ when $R$ is of type $B_{n}$ or $C_{n}$ and

$$
m_{i}=2 i-1, \quad i \in\{1, \ldots, n-1\} \quad \text { and } \quad m_{n}=n-1
$$

when $R$ is of type $D_{n}$.

\section{Remark}

(i) The integer $n-1$ appears twice in the exponents of a root system of type $D_{n}$ when $n$ is even.

(ii) The exponents $m_{i}, i=1, \ldots, n-1$ are the same for the three root systems of type $B_{n}, C_{n}$ or $D_{n}$.

As customary, we identify the lattice $P$ of weights of $G$ with a sublattice of $\left(\frac{1}{2} \mathbb{Z}\right)^{n}$. For any $\beta=\left(\beta_{1}, \ldots, \beta_{n}\right) \in P$, we set $|\beta|=\beta_{1}+\cdots+\beta_{n}$. We use for a basis of the group algebra $\mathbb{Z}\left[\mathbb{Z}^{n}\right]$, the formal exponentials $\left(e^{\beta}\right)_{\beta \in \mathbb{Z}^{n}}$ satisfying the relations $e^{\beta_{1}} e^{\beta_{2}}=e^{\beta_{1}+\beta_{2}}$. We furthermore introduce $n$ independent indeterminates $x_{1}, \ldots, x_{n}$ in order to identify $\mathbb{Z}\left[\mathbb{Z}^{n}\right]$ with the ring of polynomials $\mathbb{Z}\left[x_{1}, \ldots, x_{n}, x_{1}^{-1}, \ldots, x_{n}^{-1}\right]$ by writing $e^{\beta}=x_{1}^{\beta_{1}} \cdots x_{n}^{\beta_{n}}=x^{\beta}$ for any $\beta=\left(\beta_{1}, \ldots, \beta_{n}\right) \in \mathbb{Z}^{n}$.

Write $s_{\lambda}^{\mathfrak{g l}_{n}}$ for the Weyl character (Schur function) of the finite-dimensional $\mathfrak{g l}_{n}$-module $V^{\mathfrak{g l}} \mathfrak{l}_{n}(\lambda)$ of highest weight $\lambda$. The character ring of $G L_{n}$ is $\Lambda_{n}=$ $\mathbb{Z}\left[x_{1}, \ldots, x_{n}\right]^{\text {sym }}$ the ring of symmetric functions in $n$ variables.

For any $\lambda \in \mathcal{P}_{n}$, we denote by $s_{\lambda}^{\mathfrak{g}}$ the Weyl character of $V^{\mathfrak{g}}(\lambda)$. Let $R^{\mathfrak{g}}$ be the character ring of $G$. Then

$$
R^{\mathfrak{g}}=\mathbb{Z}\left[x_{1}, \ldots, x_{n}, x_{1}^{-1}, \ldots, x_{n}^{-1}\right]^{W^{\mathfrak{s o}} 2 n+1}
$$

is the $\mathbb{Z}$-algebra with basis $\left\{s_{\lambda}^{\mathfrak{g}} \mid \lambda \in \mathcal{P}_{n}\right\}$. 
In the sequel we will suppose $n \geq 2$ when $\mathfrak{g}=\mathfrak{s p}_{2 n}$ or $\mathfrak{s o}_{2 n+1}$ and $n \geq 4$ when $\mathfrak{g}=\mathfrak{s o}_{2 n}$.

For each Lie algebra $\mathfrak{g}=\mathfrak{s o}_{N}$ or $\mathfrak{s p}_{N}$ and any partition $v \in \mathcal{P}_{N}$, we denote by $V^{\mathfrak{g l}_{N}}(v) \downarrow_{\mathfrak{g}}^{\mathfrak{g l}_{N}}$ the restriction of $V^{\mathfrak{g l}_{N}}(v)$ to $\mathfrak{g}$. Set

$$
\begin{aligned}
& V^{\mathfrak{g l}_{N}}(\nu) \downarrow \mathfrak{s o}_{N}^{\mathfrak{g l}_{N}}=\bigoplus_{\lambda \in \mathcal{P}_{n}} V^{\mathfrak{s o}_{N}}(\lambda)^{\oplus b_{\nu, \lambda}^{\mathfrak{s o}}}
\end{aligned}
$$

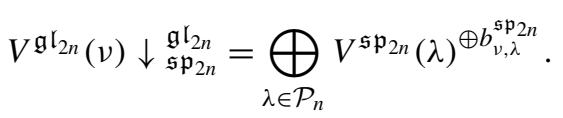

These formulas define in particular the branching coefficients $b_{v, \lambda}^{\mathfrak{s o}_{N}}$ and $b_{v, \lambda}^{\mathfrak{s p} \mathfrak{p}_{2 n}}$. The restriction map $r^{\mathfrak{g}}$ is defined by setting

$$
r^{\mathfrak{g}}:\left\{\begin{array}{l}
\mathbb{Z}\left[x_{1}, \ldots, x_{N}\right]^{\mathrm{sym}} \rightarrow R^{\mathfrak{g}} \\
s_{v}^{\mathfrak{g l}_{N}} \longmapsto \operatorname{char}\left(V^{\mathfrak{g l}_{N}}(v) \downarrow_{\mathfrak{g}}^{\mathfrak{g l}_{N}}\right)
\end{array}\right.
$$

where char $\left(V^{\mathfrak{g l}_{N}}(v) \downarrow_{\mathfrak{g}}^{\mathfrak{g l}}\right)$ is the character of the $\mathfrak{g}$-module $V^{\mathfrak{g l}_{N}}(v) \downarrow_{\mathfrak{g}}^{\mathfrak{g l}_{N}}$. We have then

$$
r^{\mathfrak{g}}\left(s_{v}^{\mathfrak{g l}_{N}}\right)= \begin{cases}s_{v}^{\mathfrak{g l}_{N}}\left(x_{1}, \ldots, x_{n}, x_{n}^{-1}, \ldots, x_{1}^{-1}\right) & \text { when } N=2 n, \\ s_{v}^{\mathfrak{g l}_{N}}\left(x_{1}, \ldots, x_{n}, 1, x_{n}^{-1}, \ldots, x_{1}^{-1}\right) & \text { when } N=2 n+1 .\end{cases}
$$

Let $\mathcal{P}_{n}^{(2)}$ and $\mathcal{P}_{n}^{(1,1)}$ be the subsets of $\mathcal{P}_{n}$ consisting of the partitions with even length rows and the partitions with even length columns, respectively. When $v \in \mathcal{P}_{n}$ we have the following formulas for the branching coefficients $b_{v, \lambda}^{\mathfrak{s o}}$ and $b_{v, \lambda}^{\mathfrak{s p}_{2 n}}$ :

Proposition 2.1.1 (see [10] appendix p. 295)

Consider $v \in \mathcal{P}_{n}$. Then:

1. $b_{\nu, \lambda}^{\mathfrak{s o}_{2 n+1}}=b_{\nu, \lambda}^{\mathfrak{s o}_{2 n}}=\sum_{\gamma \in \mathcal{P}_{n}^{(2)}} c_{\lambda, \gamma}^{\nu}$

2. $b_{\nu, \lambda}^{\mathfrak{s p}_{2 n}}=\sum_{\gamma \in \mathcal{P}_{n}^{(1,1)}} c_{\lambda, \gamma}^{v}$

where $c_{\gamma, \lambda}^{v}$ is the usual Littlewood-Richardson coefficient corresponding to the partitions $\gamma, \lambda$ and $\nu$.

Note that the equality $b_{v, \lambda}^{\mathfrak{s o}_{2 n+1}}=b_{v, \lambda}^{\mathfrak{s o}_{2 n}}$ becomes false in general when $v \notin \mathcal{P}_{n}$.

As suggested by Proposition 2.1.1, the manipulation of the Weyl characters is simplified by working with infinitely many variables. In [8], Koike and Terada have introduced a universal character ring for the classical Lie groups. This ring can be regarded as the ring $\Lambda=\mathbb{Z}\left[x_{1}, \ldots, x_{n}, \ldots\right]^{\text {sym }}$ of symmetric functions in countably many variables. It is equipped with three natural $\mathbb{Z}$-bases indexed by partitions, namely

$$
\mathcal{B}^{\mathfrak{g l}}=\left\{\mathrm{s}_{\lambda}^{\mathfrak{g l}} \mid \lambda \in \mathcal{P}\right\}, \quad \mathcal{B}^{\mathfrak{s p}}=\left\{\mathrm{s}_{\lambda}^{\mathfrak{s p}} \mid \lambda \in \mathcal{P}\right\}, \quad \mathcal{B}^{\mathfrak{s o}}=\left\{\mathrm{s}_{\lambda}^{\mathfrak{s o}} \mid \lambda \in \mathcal{P}\right\}
$$


We have in particular the decompositions:

$$
\mathbf{s}_{\mathcal{V}}^{\mathfrak{g l}}=\sum_{\lambda \in \mathcal{P}} \sum_{\gamma \in \mathcal{P}_{n}^{(2)}} c_{\lambda, \gamma}^{\nu} \mathbf{s}_{\lambda}^{\mathfrak{s o}} \quad \text { and } \quad \mathbf{s}_{\nu}^{\mathfrak{g l}}=\sum_{\lambda \in \mathcal{P}} \sum_{\gamma \in \mathcal{P}_{n}^{(1,1)}} c_{\lambda, \gamma}^{\nu} \mathbf{s}_{\lambda}^{\mathfrak{s p}}
$$

We denote by $\varphi$ the linear involution defined on $\Lambda$ by $\varphi\left(s_{\lambda}^{\mathfrak{g l} l}\right)=s_{\lambda^{\prime}}^{\mathfrak{g l}}$. Then, one has

$$
\varphi\left(\mathrm{s}_{\lambda}^{\mathfrak{s o}}\right)=\mathrm{s}_{\lambda^{\prime}}^{\mathfrak{s p}}
$$

For any positive integer $n$, denote by $\Lambda_{n}=\mathbb{Z}\left[x_{1}, \ldots, x_{n}\right]^{\text {sym }}$ the ring of symmetric functions in $n$ variables. Write

$$
\pi_{n}: \mathbb{Z}\left[x_{1}, \ldots, x_{n}, \ldots\right]^{\mathrm{sym}} \rightarrow \mathbb{Z}\left[x_{1}, \ldots, x_{n}\right]^{\mathrm{sym}}
$$

for the ring homomorphism obtained by specializing each variable $x_{i}, i>n$ at 0 . Then $\pi_{n}\left(\mathrm{~s}_{\lambda}^{\mathfrak{g l}}\right)=s_{\lambda}^{\mathfrak{g l}_{n}}$. Let $\pi^{\mathfrak{s p}_{2 n}}$ and $\pi^{\mathfrak{s o}_{N}}$ be the specialization homomorphisms defined by setting $\pi^{\mathfrak{s p} 2 n}=r^{\mathfrak{s p} p_{2 n}} \circ \pi_{2 n}$ and $\pi^{\mathfrak{s o}_{N}}=r^{\mathfrak{s o}_{N}} \circ \pi_{N}$. For any partition $\lambda \in \mathcal{P}_{n}$ one has

$$
s_{\lambda}^{\mathfrak{s p}_{2 n}}=\pi^{\mathfrak{s p}_{2 n}}\left(\mathrm{~s}_{\lambda}^{\mathfrak{s p}}\right) \quad \text { and } \quad s_{\lambda}^{\mathfrak{s o}_{N}}=\pi^{\mathfrak{s o}_{N}}\left(\mathrm{~s}_{\lambda}^{\mathfrak{s o}}\right) .
$$

We shall also need the following proposition (see [8] Corollary 2.5.3).

Proposition 2.1.2 Consider a Lie algebra $\mathfrak{g}$ of type $X_{n} \in\left\{B_{n}, C_{n}, D_{n}\right\}$. Let $\lambda \in \mathcal{P}_{r}$ and $\mu \in \mathcal{P}_{s}$. Suppose $n \geq r+s$ and set

$$
V^{\mathfrak{g}}(\lambda) \otimes V^{\mathfrak{g}}(\mu)=\bigoplus_{\nu \in \mathcal{P}_{n}} V^{\mathfrak{g}}(\nu)^{\oplus d_{\lambda, \mu}^{\nu}}
$$

Then the coefficients $d_{\lambda, \mu}^{v}$ neither depend on the rank $n$ of $\mathfrak{g}$ nor on its type $B, C$ or D.

Remark The previous proposition follows from the decompositions

$$
\mathbf{s}_{\lambda}^{\mathfrak{s p}} \times \mathbf{s}_{\mu}^{\mathfrak{s p}}=\sum_{\nu \in \mathcal{P}} d_{\lambda, \mu}^{v} \mathbf{s}_{\nu}^{\mathfrak{s p}} \quad \text { and } \quad \mathbf{s}_{\lambda}^{\mathfrak{s o}} \times \mathbf{s}_{\mu}^{\mathfrak{s o}}=\sum_{\nu \in \mathcal{P}} d_{\lambda, \mu}^{\nu} \mathbf{s}_{\nu}^{\mathfrak{s o}}
$$

for any $\lambda, \mu \in \mathcal{P}$, in the ring $\Lambda$.

\subsection{Lusztig $q$-analogues}

The $q$-analogue $\mathcal{P}_{q}^{\mathfrak{g}}$ of the Kostant partition function associated to the root system $R$ of the Lie algebra $\mathfrak{g}$ is defined by the equality

$$
\prod_{\alpha \in R^{+}} \frac{1}{1-q e^{\alpha}}=\sum_{\beta \in \mathbb{Z}^{n}} \mathcal{P}_{q}^{\mathfrak{g}}(\beta) e^{\beta}
$$


Note that $\mathcal{P}_{q}^{\mathfrak{g}}(\beta)=0$ if $\beta \notin Q^{+}$. Given $\lambda$ and $\mu$ two partitions of $\mathcal{P}_{n}$, the Lusztig $q$-analogues of weight multiplicity is the polynomial

$$
K_{\lambda, \mu}^{\mathfrak{g}}(q)=\sum_{w \in W^{\mathfrak{g}}}(-1)^{\ell(w)} \mathcal{P}_{q}^{\mathfrak{g}}(w \circ \lambda-\mu) .
$$

It follows from the Weyl character formula that $K_{\lambda, \mu}^{\mathfrak{g}}(1)$ is equal to the dimension of $V^{\mathfrak{g}}(\lambda)_{\mu}$.

\section{Theorem 2.2.1 (Lusztig [14])}

For any partitions $\lambda, \mu \in \mathcal{P}_{n}$, the polynomial $K_{\lambda, \mu}^{\mathfrak{g}}(q)$ has nonnegative integer coefficients.

We write

$$
K_{\lambda, \mu}^{\mathfrak{g}}(q)=\sum_{k \geq 0} K_{\lambda, \mu}^{\mathfrak{g}, k} q^{k}
$$

Then

$$
K_{\lambda, \mu}^{\mathfrak{g}, k}(q)=\sum_{w \in W^{\mathfrak{g}}}(-1)^{\ell(w)} \mathcal{P}^{k}(w \circ \lambda-\mu)
$$

where for any $\beta \in \mathbb{Z}^{n}, \mathcal{P}^{k}(\beta)$ is the number of ways of decomposing $\beta$ as a sum of $k$ positive roots.

Remark One easily verifies that $K_{\lambda, \mu}^{\mathfrak{g}}(q) \neq 0$ only if $\lambda \geq \mu$. Moreover, when $|\mu|=|\lambda|$, one has $K_{\lambda, \mu}^{\mathfrak{g}}(q)=K_{\lambda, \mu}^{\mathfrak{g l}_{n}}(q)$ where $K_{\lambda, \mu}^{\mathfrak{g l} l_{n}}(q)$ is the Kostka polynomial associated to $(\lambda, \mu)$, i.e. the Lusztig $q$-analogue associated to the partitions $\lambda, \mu$ for the root system $A_{n-1}$.

We also introduce the Hall-Littlewood polynomials $Q_{\mu}^{\prime \mathfrak{g}}, \mu \in \mathcal{P}_{n}$ defined by

$$
Q_{\mu}^{\prime \mathfrak{g}}=\sum_{\lambda \in \mathcal{P}_{n}} K_{\lambda, \mu}^{\mathfrak{g}}(q) s_{\lambda}^{\mathfrak{g}}
$$

\subsection{The symmetric algebra $S(\mathfrak{g})$}

Considered as a $G$-module, $\mathfrak{g}$ is irreducible and we have

$$
\left\{\begin{array}{l}
\mathfrak{s o}_{2 n+1} \simeq V^{\mathfrak{s o}_{2 n+1}}(1,1) \quad \text { and } \quad \operatorname{dim}\left(\mathfrak{s o}_{2 n+1}\right)=n(2 n+1) \\
\mathfrak{s p}_{2 n} \simeq V^{\mathfrak{s p}_{2 n}}(2) \quad \text { and } \quad \operatorname{dim}\left(\mathfrak{s p}_{2 n}\right)=n(2 n+1) \\
\mathfrak{s o}_{2 n} \simeq V^{\mathfrak{s o}_{2 n}}(1,1) \quad \text { and } \quad \operatorname{dim}\left(\mathfrak{s o}_{2 n}\right)=n(2 n-1)
\end{array}\right.
$$

Let $S(\mathfrak{g})$ be the symmetric algebra of $\mathfrak{g}$ and set

$$
S(\mathfrak{g})=\bigoplus_{k \geq 0} S^{k}(\mathfrak{g})
$$


where $S^{k}(\mathfrak{g})$ is the $k$-th symmetric power of $\mathfrak{g}$. By Proposition 2.1.1 and (13), we have

$$
\mathfrak{s o}_{N} \simeq V^{\mathfrak{g l}_{N}}(1,1) \downarrow_{\mathfrak{s o}_{N}}^{\mathfrak{g l}_{N}} \quad \text { and } \quad \mathfrak{s p}_{2 n} \simeq V^{\mathfrak{g l}_{2 n}}(2) \downarrow_{\mathfrak{s} \mathfrak{p}_{2 n}}^{\mathfrak{g l} \mathfrak{l}_{2 n}}
$$

This implies the following isomorphisms

$$
S^{k}\left(\mathfrak{s o}_{N}\right) \simeq S^{k}\left(V^{\mathfrak{s o}_{N}}(1,1)\right) \downarrow_{\mathfrak{s} \mathfrak{o}_{N}}^{\mathfrak{g l}} \quad \text { and } \quad S^{k}\left(\mathfrak{s p}_{2 n}\right) \simeq S^{k}\left(V^{\mathfrak{s p}_{2 n}}(2)\right) \downarrow_{\mathfrak{s p}_{2 n}}^{\mathfrak{g l}_{2 n}}
$$

for any nonnegative integer $k$.

Example 2.3.1 By using the Weyl dimension formula (see [4] page 303), one easily obtains the decompositions

$$
S^{2}\left(V^{\mathfrak{g l}_{N}}(1,1)\right) \simeq V^{\mathfrak{g l}_{N}}(1,1,1,1) \oplus V^{\mathfrak{g l}_{N}}(2,2)
$$

and

$$
S^{2}\left(V^{\mathfrak{g l}_{2 n}}(2)\right) \simeq V^{\mathfrak{g l}_{2 n}}(4) \oplus V^{\mathfrak{g l}_{2 n}}(2,2) .
$$

Hence by (14) and Proposition 2.1.1, this gives

$$
S^{2}(\mathfrak{g}) \simeq V^{\mathfrak{g}}(1,1,1,1) \oplus V^{\mathfrak{g}}(2,2) \oplus V^{\mathfrak{g}}(2,0) \oplus V^{\mathfrak{g}}(\emptyset) \text { for } \mathfrak{g}=\mathfrak{s o}{ }_{N}
$$

and

$$
S^{2}\left(\mathfrak{s p}_{2 n}\right) \simeq V^{\mathfrak{s p}_{2 n}}(4) \oplus V^{\mathfrak{s p}} \mathfrak{p}_{2 n}(2,2) \oplus V^{\mathfrak{s p}_{2 n}}(1,1) \oplus V^{\mathfrak{s p}_{2 n}}(\emptyset)
$$

Remark By the previous formulas, the multiplicities appearing in the decomposition of the square symmetric power of the Lie algebra $\mathfrak{g}$ of type $X_{n} \in\left\{B_{n}, C_{n}, D_{n}\right\}$ do not depend on its rank providing $n \geq 2$. We give in Proposition 3.1.1, the general explicit decomposition of $S^{k}(\mathfrak{g})$ into its irreducible components.

\section{Graded characters}

\subsection{Graded character of the symmetric algebra}

Let $V$ be a $G$ or $G L_{n}$-module. For any nonnegative integer $k$, write $S^{k}(V)$ for the $k$-th symmetric power of $V$ and set $S(V)=\oplus_{k \geq 0} S^{k}(V)$. Then $S^{k}(V)$ and $S(V)$ are also $G$-modules. The graded character of $S(V)$ is defined by

$$
\operatorname{char}_{q}(S(V))=\sum_{k \geq 0} \operatorname{char}\left(S^{k}(V)\right) q^{k} .
$$

Denote by $\mathcal{W}(V)$ the collection of weights of the module $V$ counted with their multiplicities. Then we have

$$
\operatorname{char}_{q}(S(V))=\prod_{\beta \in \mathcal{W}(V)} \frac{1}{1-q e^{\beta}} .
$$


The weights of the Lie algebra $\mathfrak{g}$ of rank $n$ considered as a $G$-module are such that

$$
\mathcal{W}(\mathfrak{g})=\{\alpha \in R, \underbrace{0, \ldots, 0}_{n \text { times }}\} .
$$

Thus the graded character $\operatorname{char}_{q}(S(\mathfrak{g}))$ of $S(\mathfrak{g})$ verifies

$$
\operatorname{char}_{q}(S(\mathfrak{g}))=\frac{1}{(1-q)^{n}} \prod_{\alpha \in R} \frac{1}{1-q x^{\alpha}}
$$

Proposition 3.1.1 For any nonnegative integer $k$, we have

$$
\begin{aligned}
& \operatorname{char}\left(S^{k}\left(\mathfrak{s o}_{N}\right)\right)=\sum_{\lambda \in \mathcal{P}_{n}} \sum_{\nu \in \mathcal{P}_{N}^{(1,1)}(2 k)} b_{\nu, \lambda}^{\mathfrak{s o}_{N}} s_{\lambda}^{\mathfrak{s o}_{N}}, \\
& \operatorname{char}\left(S^{k}\left(\mathfrak{s p}_{2 n}\right)\right)=\sum_{\lambda \in \mathcal{P}_{n}} \sum_{\nu \in \mathcal{P}_{2 n}^{(2)}(2 k)} b_{\nu, \lambda}^{\mathfrak{s p}_{2 n}} s_{\lambda}^{\mathfrak{s p}_{2 n}},
\end{aligned}
$$

where $b_{v, \lambda}^{\mathfrak{s o}_{N}}$ and $b_{v, \lambda}^{\mathfrak{s p} \mathfrak{p}_{2 n}}$ are the branching coefficients defined in (7).

Proof Suppose first $\mathfrak{g}=\mathfrak{s p}_{2 n}$. Recall the classical identity

$$
\prod_{1 \leq i \leq j \leq 2 n} \frac{1}{1-x_{i} x_{j}}=\sum_{\nu \in \mathcal{P}_{2 n}^{(2)}} s_{v}^{\mathfrak{g l} \mathfrak{l}_{2 n}}
$$

due to Littlewood. It immediately implies the decomposition

$$
\prod_{1 \leq i \leq j \leq 2 n} \frac{1}{1-q x_{i} x_{j}}=\sum_{\nu \in \mathcal{P}_{2 n}^{(2)}} q^{\frac{|\nu|}{2}} s_{\nu}^{\mathfrak{g l}_{2 n}}=\sum_{k \geq 0} \sum_{\nu \in \mathcal{P}_{2 n}^{(2)}(2 k)} s_{\nu}^{\mathfrak{g l}_{2 n}} q^{k} .
$$

By applying the restriction map $r^{\mathfrak{s p}_{2 n}}$, this gives

$$
\begin{aligned}
& \frac{1}{(1-q)^{n}} \prod_{1 \leq i<j \leq n} \frac{1}{1-q \frac{x_{i}}{x_{j}}} \frac{1}{1-q \frac{x_{j}}{x_{i}}} \prod_{1 \leq r \leq s \leq n} \frac{1}{1-q x_{r} x_{s}} \frac{1}{1-q \frac{1}{x_{r} x_{s}}} \\
& =\sum_{k \geq 0} \sum_{v \in \mathcal{P}_{2 n}^{(2)}(2 k)} s_{v}^{\mathfrak{g} l_{2 n}}\left(x_{1}, \ldots, x_{n}, x_{n}^{-1}, \ldots, x_{1}^{-1}\right) q^{k} .
\end{aligned}
$$

From (7), this can be rewritten in the form

$$
\operatorname{char}_{q}\left(S\left(\mathfrak{s p}_{2 n}\right)\right)=\frac{1}{(1-q)^{n}} \prod_{\alpha \in R} \frac{1}{1-q x^{\alpha}}=\sum_{k \geq 0} \sum_{\lambda \in \mathcal{P}_{n}} \sum_{\nu \in \mathcal{P}_{2 n}^{(2)}(2 k)} b_{\nu, \lambda}^{\mathfrak{s p}_{2 n}} s_{\lambda}^{\mathfrak{s p}_{2 n}} q^{k}
$$

which gives the desired identity by considering the coefficient in $q^{k}$. 
When $\mathfrak{g}=\mathfrak{s o}_{2 n+1}$ or $\mathfrak{g}=\mathfrak{s o}_{2 n}$, one uses the identity

$$
\prod_{1 \leq i<j \leq 2 n} \frac{1}{1-q x_{i} x_{j}}=\sum_{\nu \in \mathcal{P}_{2 n}^{(1,1)}} q^{\frac{|\nu|}{2}} s_{\nu}^{\mathfrak{g l} \mathfrak{l}_{2 n}}=\sum_{k \geq 0} \sum_{\nu \in \mathcal{P}_{2 n}^{(1,1)}(2 k)} s_{v}^{\mathfrak{g l}_{2 n}} q^{k}
$$

and our result follows by similar arguments.

In the sequel, we set

$$
m_{k, \lambda}^{\mathfrak{s o}_{N}}=\sum_{\nu \in \mathcal{P}_{N}^{(1,1)}(2 k)} b_{\nu, \lambda}^{\mathfrak{s o}_{N}} \quad \text { and } \quad m_{k, \lambda}^{\mathfrak{s p}_{2 n}}=\sum_{\nu \in \mathcal{P}_{2 n}^{(2)}(2 k)} b_{\nu, \lambda}^{\mathfrak{s p}_{2 n}}
$$

Thus we have $\operatorname{char}\left(S^{k}\left(\mathfrak{s o}_{N}\right)\right)=\sum_{\lambda \in \mathcal{P}_{n}} m_{k, \lambda}^{\mathfrak{s o}_{N}} s_{\lambda}^{\mathfrak{s o}_{N}}$ and $\operatorname{char}\left(S^{k}\left(\mathfrak{s p}_{2 n}\right)\right)=$ $\sum_{\lambda \in \mathcal{P}_{n}} m_{k, \lambda}^{\mathfrak{s p}_{2 n}} s_{\lambda}^{\mathfrak{s p}_{2 n}}$

3.2 Universal graded characters $\operatorname{char}_{q}(S(\mathfrak{s p}))$ and $\operatorname{char}_{q}(S(\mathfrak{s o}))$

Proposition 3.2.1 Consider a nonnegative integer $k$ and suppose $n \geq 2 k$. For any $\lambda \in \mathcal{P}$, we have the identities

$$
\begin{aligned}
m_{k, \lambda}^{\mathfrak{s o}_{2 n+1}} & =m_{k, \lambda}^{\mathfrak{s o}_{2 n}}=\sum_{\nu \in \mathcal{P}^{(1,1)}(2 k)} \sum_{\gamma \in \mathcal{P}^{(2)}} c_{\lambda, \gamma}^{\nu}, \\
m_{k, \lambda}^{\mathfrak{s p}_{2 n}} & =\sum_{\nu \in \mathcal{P}^{(2)}(2 k)} \sum_{\gamma \in \mathcal{P}^{(1,1)}} c_{\lambda, \gamma}^{\nu} .
\end{aligned}
$$

In particular, the multiplicities $m_{k, \lambda}^{\mathfrak{s p}_{2 n}}, m_{k, \lambda}^{\mathfrak{s o}_{2 n+1}}, m_{k, \lambda}^{\mathfrak{s o}_{2 n}}$ do not depend on $n$.

Proof For any $v \in \mathcal{P}_{N}(2 k)$ we have $v \in \mathcal{P}_{N}[n]$ since $n \geq 2 k$. In particular $v \in \mathcal{P}_{n}$. We can thus deduce from Propositions 2.1.1 and 3.1.1 the decompositions

$$
m_{k, \lambda}^{\mathfrak{s o}_{N}}=\sum_{\nu \in \mathcal{P}_{n}^{(1,1)}(2 k)} \sum_{\gamma \in \mathcal{P}_{2 k}^{(2)}} c_{\lambda, \gamma}^{\nu} \text { and } m_{k, \lambda}^{\mathfrak{s p}_{2 n}}=\sum_{\nu \in \mathcal{P}_{n}^{(2)}(2 k)} \sum_{\gamma \in \mathcal{P}_{2 k}^{(1,1)}} c_{\lambda, \gamma}^{\nu}
$$

Since $c_{\lambda, \gamma}^{\nu}=0$ when $|\lambda|+|\gamma| \neq 2 k$ (so $|\lambda| \leq 2 k \leq n$ and $\left.|\gamma| \leq n\right), m_{k, \lambda}^{\mathfrak{s o}_{N}}$ and $m_{k, \lambda}^{\mathfrak{s p}_{2 n}}$ can be rewritten as in (16) and thus, do not depend on $n$.

We set

$$
\begin{aligned}
m_{k, \lambda}^{\mathfrak{s o}} & =\lim _{n \rightarrow \infty} m_{k, \lambda}^{\mathfrak{s o}_{2 n+1}}=\sum_{\nu \in \mathcal{P}^{(1,1)}(2 k)} \sum_{\gamma \in \mathcal{P}^{(1,1)}} c_{\lambda, \gamma}^{\nu} \quad \text { and } \quad m_{k, \lambda}^{\mathfrak{s p}}=\lim _{n \rightarrow \infty} m_{k, \lambda}^{\mathfrak{s p}_{2 n}} \\
& =\sum_{\nu \in \mathcal{P}^{(2)}(2 k)} \sum_{\gamma \in \mathcal{P}^{(2)}} c_{\lambda, \gamma}^{\nu} .
\end{aligned}
$$


Lemma 3.2.2 For any nonnegative integer $k$ and any partition $\lambda$, we have

1. $m_{k, \lambda}^{\mathfrak{s p}}=m_{k, \lambda^{\prime}}^{\mathfrak{s o}}$

2. $m_{k, \lambda}^{\mathfrak{s p}}=m_{k, \lambda}^{\mathfrak{s o}}=0$ if $|\lambda|>2 k$

where $m_{k, \lambda}^{\mathfrak{s p}}$ and $m_{k, \lambda^{\prime}}^{\mathfrak{s o}}$ are the multiplicities defined in (17).

Proof Write $\iota$ for the bijective map defined on $\mathcal{P}$ by $\lambda \longmapsto \lambda^{\prime}$. We then have $\iota\left(\mathcal{P}^{(2)}\right)=\mathcal{P}^{(1,1)}$ and $\iota\left(\mathcal{P}^{(2)}(2 k)\right)=\mathcal{P}^{(1,1)}(2 k)$. Moreover $c_{\lambda, \gamma}^{\nu}=c_{\lambda^{\prime}, \gamma^{\prime}}^{\nu^{\prime}}$ for any partitions $\lambda, \gamma, v$. This implies assertion 1 from the definition (17) of $m_{k, \lambda}^{\mathfrak{s p}}$ and $m_{k, \lambda^{\prime}}^{\mathfrak{s o}}$.

Recall that $c_{\lambda, \gamma}^{\nu}=0$ when $|\nu| \neq|\lambda|+|\gamma|$. Since $|\nu|=2 k$ in the equalities of (17), we have $m_{k, \lambda}^{\mathfrak{s p}}=m_{k, \lambda}^{\mathfrak{s o}}=0$ if $|\lambda|>2 k$.

We define the universal graded characters $\operatorname{char}_{q}(S(\mathfrak{s p}))$ and $\operatorname{char}_{q}(S(\mathfrak{s o}))$ by setting

$$
\operatorname{char}_{q}(\mathrm{~S}(\mathfrak{s p}))=\prod_{1 \leq i \leq j} \frac{1}{1-q x_{i} x_{j}} \quad \text { and } \quad \operatorname{char}_{q}(\mathrm{~S}(\mathfrak{s p}))=\prod_{1 \leq i<j} \frac{1}{1-q x_{i} x_{j}}
$$

Note that $\operatorname{char}_{q}(S(\mathfrak{s p}))$ and $\operatorname{char}_{q}(S(\mathfrak{s o}))$ belong to the ring $\Lambda[[q]]$ of formal series with coefficients in $\Lambda$.

For any $F=\sum_{k \geq 0} c_{k} q^{k}$ in $\Lambda[[q]]$, the specialization homomorphisms $\pi^{\mathfrak{s p} 2 n}$, $\pi^{\mathfrak{s o}_{2 n+1}}$ and $\pi^{\mathfrak{s o}_{2 n}}$ are then defined by setting

$$
\pi^{\mathfrak{g}}(F)=\sum_{k \geq 0} \pi^{\mathfrak{g}}\left(c_{k}\right) q^{k}
$$

By (15) and (18), we then have

$$
\begin{aligned}
& \pi^{\mathfrak{s o}}\left(\operatorname{char}_{q}(\mathrm{~S}(\mathfrak{s o}))\right)=\operatorname{char}_{q}\left(S\left(\mathfrak{s o}_{N}\right)\right), \\
& \pi^{\mathfrak{s} \mathfrak{p}_{2 n}}\left(\operatorname{char}_{q}(S(\mathfrak{s p}))\right)=\operatorname{char}_{q}\left(S\left(\mathfrak{s p}_{2 n}\right)\right) .
\end{aligned}
$$

Similarly, the linear involution $\varphi$ (see (10)) is defined on $\Lambda[[q]]$ by

$$
\varphi(F)=\sum_{k \geq 0} \varphi\left(c_{k}\right) q^{k}
$$

Proposition 3.2.3 We have the decompositions

$$
\begin{aligned}
& \operatorname{char}_{q}(\mathrm{~S}(\mathfrak{s o}))=\sum_{k \geq 0} \sum_{\lambda \in \mathcal{P}} m_{k, \lambda}^{\mathfrak{s o}} \mathbf{S}_{\lambda}^{\mathfrak{s o}} q^{k}, \\
& \operatorname{char}_{q}(\mathrm{~S}(\mathfrak{s p}))=\sum_{k \geq 0} \sum_{\lambda \in \mathcal{P}} m_{k, \lambda}^{\mathfrak{s p}} \mathbf{S}_{\lambda}^{\mathfrak{s p}} q^{k} .
\end{aligned}
$$


Proof One can write

$$
\begin{aligned}
& \operatorname{char}_{q}(\mathrm{~S}(\mathfrak{s o}))=\prod_{1 \leq i<j} \frac{1}{1-q x_{i} x_{j}}=\sum_{k \geq 0} \sum_{\nu \in \mathcal{P}^{(1,1)}(2 k)} \mathrm{s}_{v}^{\mathfrak{g l}} q^{k}=\sum_{k \geq 0} \sum_{\lambda \in \mathcal{P}} m_{k, \lambda}^{\mathfrak{s o}} \mathrm{s}_{\lambda}^{\mathfrak{s o}} q^{k} \\
& \operatorname{char}_{q}(\mathrm{~S}(\mathfrak{s p}))=\prod_{1 \leq i \leq j} \frac{1}{1-q x_{i} x_{j}}=\sum_{k \geq 0} \sum_{\nu \in \mathcal{P}^{(2)}(2 k)} \mathrm{s}_{\nu}^{\mathfrak{g l}} q^{k}=\sum_{k \geq 0} \sum_{\lambda \in \mathcal{P}} m_{k, \lambda}^{\mathfrak{s p}} \mathrm{s}_{\lambda}^{\mathfrak{s p}} q^{k}
\end{aligned}
$$

where the rightmost equalities follow from (9).

By using 1 of Lemma 3.2.2, one derives the following corollary:

Corollary 3.2.4 We have $\varphi\left(\operatorname{char}_{q}(S(\mathfrak{s o}))\right)=\operatorname{char}_{q}(S(\mathfrak{s p}))$.

Remark By 2 of Lemma 3.2.2, one has

$$
\operatorname{char}\left(S^{k}(\mathfrak{s o})\right)=\sum_{\lambda \in \mathcal{P}[2 k]} m_{k, \lambda}^{\mathfrak{s o}} \mathrm{S}_{\lambda}^{\mathfrak{s o}} \text { and } \operatorname{char}\left(S^{k}(\mathfrak{s p})\right)=\sum_{\lambda \in \mathcal{P}[2 k]} m_{k, \lambda}^{\mathfrak{s p}} \mathrm{S}_{\lambda}^{\mathfrak{s p}}
$$

where $\mathcal{P}[2 k]$ is the set of partitions $\lambda$ such that $|\lambda| \leq 2 k$.

\subsection{Universal graded character for harmonic polynomials}

Let $\mathfrak{g}$ be a Lie algebra of type $X_{n} \in\left\{B_{n}, C_{n}, D_{n}\right\}$. Since the symmetric algebra $S(\mathfrak{g})$ can be regarded as a $G$-module, one can consider

$$
S(\mathfrak{g})^{G}=\{x \in S(\mathfrak{g}) \mid g \cdot x=x \text { for any } g \in G\},
$$

the ring of the $G$-invariants in $S(\mathfrak{g})$. By a classical theorem of Kostant [8] we have

$$
S(\mathfrak{g})=H(\mathfrak{g}) \otimes S(\mathfrak{g})^{G}
$$

where $H(\mathfrak{g})$ is the ring of $G$-harmonic polynomials. The $\operatorname{ring} S(\mathfrak{g})^{G}$ is generated by algebraically independent homogeneous polynomials of degrees $d_{i}=m_{i}+1$ and the graded character of $S(\mathfrak{g})^{G}$ considered as a $G$-module verifies

$$
\operatorname{char}_{q}\left(S(\mathfrak{g})^{G}\right)=\prod_{i=1}^{n} \frac{1}{1-q^{d_{i}}}
$$

By (24) the graded character of $H(\mathfrak{g})$ can be written

$$
\operatorname{char}_{q}(H(\mathfrak{g}))=\frac{\operatorname{char}_{q}(S(\mathfrak{g}))}{\operatorname{char}_{q}\left(S(\mathfrak{g})^{G}\right)}=\prod_{i=1}^{n}\left(1-q^{d_{i}}\right) \operatorname{char}_{q}(S(\mathfrak{g}))=\sum_{k \geq 0} \operatorname{char}\left(H^{k}(\mathfrak{g})\right) q^{k}
$$



ting

We define the universal graded characters $\operatorname{char}_{q}(H(\mathfrak{s p}))$ and $\operatorname{char}_{q}(H(\mathfrak{s o}))$ by set-

$$
\begin{aligned}
& \operatorname{char}_{q}(\mathrm{H}(\mathfrak{s o}))=\prod_{i \geq 1}\left(1-q^{2 i}\right) \operatorname{char}_{q}(\mathrm{~S}(\mathfrak{s o}))=\prod_{i \geq 1}\left(1-q^{2 i}\right) \prod_{1 \leq i<j} \frac{1}{1-q x_{i} x_{j}}, \\
& \operatorname{char}_{q}(\mathrm{H}(\mathfrak{s p}))=\prod_{i \geq 1}\left(1-q^{2 i}\right) \operatorname{char}_{q}(\mathrm{~S}(\mathfrak{s p}))=\prod_{i \geq 1}\left(1-q^{2 i}\right) \prod_{1 \leq i \leq j} \frac{1}{1-q x_{i} x_{j}} .
\end{aligned}
$$

The universal characters $\operatorname{char}_{q}(S(\mathfrak{s p}))$ and $\operatorname{char}_{q}(S(\mathfrak{s o}))$ belong to $\Lambda[[q]]$. Moreover $\prod_{i \geq 1}\left(1-q^{2 i}\right) \in \Lambda[[q]]$ since it is a formal series in $q$ with integer coefficients. Hence $\operatorname{char}_{q}(\mathrm{H}(\mathfrak{s p}))$ and $\operatorname{char}_{q}(\mathrm{H}(\mathfrak{s o}))$ also belong to $\Lambda[[q]]$. We set

$$
\begin{aligned}
\operatorname{char}_{q}(\mathrm{H}(\mathfrak{s p})) & =\sum_{k \geq 0} \sum_{\lambda \in \mathcal{P}} K_{\lambda, \emptyset}^{\mathfrak{s p}, k} \mathrm{~s}_{\lambda}^{\mathfrak{s p}} q^{k}, \\
\operatorname{char}_{q}(\mathrm{H}(\mathfrak{s o})) & =\sum_{k \geq 0} \sum_{\lambda \in \mathcal{P}} K_{\lambda, \emptyset}^{\mathfrak{s o}, k} \mathrm{~s}_{\lambda}^{\mathfrak{s o}} q^{k} .
\end{aligned}
$$

Lemma 3.3.1 For any nonnegative integer $k$, we have

$$
\operatorname{char}\left(\mathrm{H}^{k}(\mathfrak{s o})\right)=\sum_{\lambda \in \mathcal{P}[2 k]} K_{\lambda, \emptyset}^{\mathfrak{s o}, k} \mathrm{~s}_{\lambda}^{\mathfrak{s o}} \text { and } \operatorname{char}\left(\mathrm{H}^{k}(\mathfrak{s p})\right)=\sum_{\lambda \in \mathcal{P}[2 k]} K_{\lambda, \emptyset}^{\mathfrak{s p}, k} \mathrm{~s}_{\lambda}^{\mathfrak{s p}}
$$

Moreover, for any nonnegative integer $n \geq 2 k$ we have

$$
\begin{aligned}
& \pi^{\mathfrak{s o}_{N}}\left(\operatorname{char}\left(H^{k}(\mathfrak{s o})\right)\right)=\operatorname{char}\left(H^{k}\left(\mathfrak{s o}_{N}\right)\right), \\
& \pi^{\mathfrak{s p}_{2 n}}\left(\operatorname{char}\left(\mathrm{H}^{k}(\mathfrak{s p})\right)\right)=\operatorname{char}\left(H^{k}\left(\mathfrak{s p}_{2 n}\right)\right) .
\end{aligned}
$$

Proof By definition of the coefficients $K_{\lambda, \emptyset}^{\mathfrak{s o}, k}$, we have

$$
\operatorname{char}\left(\mathrm{H}^{k}(\mathfrak{s o})\right)=\sum_{\lambda \in \mathcal{P}} K_{\lambda, \emptyset}^{\mathfrak{s o}, k} \mathrm{~s}_{\lambda}^{\mathfrak{s o}}
$$

Write [k/2] for the largest integer which does not exceed $k / 2$. Then by (26), $K_{\lambda, \emptyset}^{\mathfrak{s o}, k}$ is the coefficient in $q^{k} \mathrm{~S}_{\lambda}^{\mathfrak{s o}}$ appearing in the expansion of

$$
\psi_{k}=\prod_{i=1}^{[k / 2]}\left(1-q^{2 i}\right) \sum_{a=0}^{k} q^{a} \operatorname{char}\left(S^{a}(\mathfrak{s o})\right)
$$

on the basis $\left\{q^{k} \mathrm{~s}_{\lambda}^{\mathfrak{s o}} \mid k \in \mathbb{N}, \lambda \in \mathcal{P}\right\}$ of $\Lambda[q]$. Indeed, for any $i>[k / 2]$, we have $2 i>k$. By (23),

$$
\operatorname{char}\left(S^{a}(\mathfrak{s o})\right)=\sum_{\lambda \in \mathcal{P}[2 a]} m_{a, \lambda}^{\mathfrak{s o}} \mathrm{S}_{\lambda}^{\mathfrak{s o}}
$$


for any nonnegative integer $a$. Since the integers $a$ appearing in (30) are less or equal to $k$, we have $m_{a, \lambda}^{\mathfrak{s o}}=0$ for any partition $\lambda$ such that $|\lambda|>2 k$, by assertion 2 of Lemma 3.2.2. Hence, the coefficient of $q^{b} s_{\lambda}^{\mathfrak{s o}}$ in $\psi_{k}$ is equal to 0 when $|\lambda|>2 k$. This permits to consider only the partitions of $\mathcal{P}[2 k]$ in the sum (29) and yields (27). The proof is the same for $\operatorname{char}\left(\mathrm{H}^{k}(\mathfrak{s p})\right)$.

We have seen that $\operatorname{char}\left(\mathrm{H}^{k}(\mathfrak{s o})\right)$ is the coefficient in $q^{k}$ of $\psi_{k}$. Now for any rank $n \geq 2 k, \operatorname{char}\left(H^{k}\left(\mathfrak{s o}_{N}\right)\right)$ is the coefficient of $q^{k}$ in

$$
\phi_{k}=\prod_{i=1}^{[k / 2]}\left(1-q^{2 i}\right) \sum_{a=0}^{k} q^{a} \operatorname{char}\left(S^{a}\left(\mathfrak{s o}_{N}\right)\right) .
$$

Indeed we have

$$
\operatorname{char}_{q}\left(H\left(\mathfrak{s o}_{N}\right)\right)=\prod_{i=1}^{n}\left(1-q^{d_{i}}\right) \operatorname{char}_{q}\left(S\left(\mathfrak{s o}_{N}\right)\right)
$$

and $d_{i}>k$ for any $i>[k / 2]$. By (20), we know that $\pi^{\mathfrak{s o}_{N}}\left(\operatorname{char}\left(\mathrm{S}^{a}(\mathfrak{s o})\right)=\right.$ $\operatorname{char}\left(S^{a}\left(\mathfrak{s o}_{N}\right)\right)$. Thus this gives the equality $\pi^{\mathfrak{s o}_{N}}\left(\psi_{k}\right)=\phi_{k}$. Hence we have $\pi^{\mathfrak{s o}_{N}}\left(\operatorname{char}\left(\mathrm{H}^{k}(\mathfrak{s o})\right)\right)=\operatorname{char}\left(H^{k}\left(\mathfrak{s o}_{N}\right)\right)$.

The proof is similar for $\operatorname{char}\left(\mathrm{H}^{k}(\mathfrak{s p})\right)$.

Remark Since (28) only holds for $n \geq 2 k$, we have

$$
\begin{aligned}
& \pi^{\mathfrak{s o}_{N}}\left(\operatorname{char}_{q}(\mathrm{H}(\mathfrak{s o}))\right) \neq \operatorname{char}_{q}\left(H\left(\mathfrak{s o}_{N}\right)\right), \\
& \pi^{\mathfrak{s p}_{2 n}}\left(\operatorname{char}_{q}(\mathrm{H}(\mathfrak{s p}))\right) \neq \operatorname{char}_{q}\left(H\left(\mathfrak{s p}_{2 n}\right)\right)
\end{aligned}
$$

i.e. there do not exist identities analogous to (20) for $\operatorname{char}_{q}(\mathrm{H}(\mathfrak{s o}))$ and $\operatorname{char}_{q}(\mathrm{H}(\mathfrak{s p}))$.

\section{Stabilization of the coefficients $K_{\lambda, \mu}^{\mathfrak{g}, k}$}

\subsection{Stabilization of the coefficients $K_{\lambda, \emptyset}^{\mathfrak{g}, k}$}

The theorem below shows that the coefficients of the expansion of $\operatorname{char}_{q}(H(\mathfrak{g}))$ on the basis of Weyl characters are the Lusztig $q$-analogues associated to the zero weight (i.e. $\mu=\emptyset$ ).

Theorem 4.1.1 (Hesselink [5]) Let $\mathfrak{g}$ be a classical Lie algebra of rank $n$. We have

$$
\operatorname{char}_{q}(H(\mathfrak{g}))=\sum_{\lambda \in \mathcal{P}_{n}} K_{\lambda, \emptyset}^{\mathfrak{g}}(q) s_{\lambda}^{\mathfrak{g}}=\sum_{k \geq 0} \sum_{\lambda \in \mathcal{P}_{n}} K_{\lambda, \emptyset}^{\mathfrak{g}, k} q^{k} s_{\lambda}^{\mathfrak{g}} .
$$

The multiplicity of $V^{\mathfrak{g}}(\lambda)$ in the decomposition of $H^{k}(\mathfrak{g})$ in its irreducible components is equal to $K_{\lambda, \emptyset}^{\mathfrak{g}, k}$. 
Now fix a nonnegative integer $k$ and choose a rank $n \geq 2 k$. The partitions $\lambda$ appearing in (27) verify $|\lambda| \leq 2 k$. Hence they belong to $\mathcal{P}_{n}$ for $n \geq 2 k$. Since $\pi^{\mathfrak{s o}_{N}}\left(s_{\lambda}^{\mathfrak{s o}}\right)=s_{\lambda}^{\mathfrak{s o}_{N}}$ and $\pi^{\mathfrak{s p}_{2 n}}\left(s_{\lambda}^{\mathfrak{s p}}\right)=s_{\lambda}^{\mathfrak{s p}_{2 n}}$ for any $\lambda \in \mathcal{P}_{n}$ this gives

$$
\pi^{\mathfrak{s o} \mathfrak{o}_{N}}\left(\operatorname{char}\left(\mathrm{H}^{k}(\mathfrak{s o})\right)\right)=\sum_{\lambda \in \mathcal{P}_{n}} K_{\lambda, \emptyset}^{\mathfrak{s o}, k} s_{\lambda}^{\mathfrak{s o} \mathfrak{o}_{N}}
$$

and

$$
\pi^{\mathfrak{s p}_{2 n}}\left(\operatorname{char}\left(\mathrm{H}^{k}(\mathfrak{s p})\right)\right)=\sum_{\lambda \in \mathcal{P}_{n}} K_{\lambda, \emptyset}^{\mathfrak{s p}, k} s_{\lambda}^{\mathfrak{s p} 2 n}
$$

By using (28), one obtains

$$
\operatorname{char}\left(H^{k}\left(\mathfrak{s o}_{N}\right)\right)=\sum_{\lambda \in \mathcal{P}_{n}} K_{\lambda, \emptyset}^{\mathfrak{s o}, k} s_{\lambda}^{\mathfrak{s o}_{N}} \text { and } \operatorname{char}\left(H^{k}\left(\mathfrak{s p}_{2 n}\right)\right)=\sum_{\lambda \in \mathcal{P}_{n}} K_{\lambda, \emptyset}^{\mathfrak{s p}, k} s_{\lambda}^{\mathfrak{s p}_{2 n}}
$$

We can now state the stabilization result for the coefficients $K_{\lambda, \emptyset}^{\mathfrak{g}, k}$.

Proposition 4.1.2 Let $m, k$ be nonnegative integers. Consider $\lambda \in \mathcal{P}_{m}$ and $\mathfrak{g}$ a Lie algebra of type $X_{n} \in\left\{B_{n}, C_{n}, D_{n}\right\}$. Suppose $n \geq 2 k$, then

$$
K_{\lambda, \emptyset}^{\mathfrak{s p} \mathfrak{p}_{2 n}, k}=K_{\lambda, \emptyset}^{\mathfrak{s p}, k} \quad \text { and } \quad K_{\lambda, \emptyset}^{\mathfrak{s o} 0_{2 n+1}, k}=K_{\lambda, \emptyset}^{\mathfrak{s o} \mathfrak{o}_{2 n}, k}=K_{\lambda, \emptyset}^{\mathfrak{s o}, k}
$$

In particular the coefficients $K_{\lambda, \emptyset}^{\mathfrak{s p}_{2 n}, k}, K_{\lambda, \emptyset}^{\mathfrak{s o}_{2 n+1}, k}$ and $K_{\lambda, \emptyset}^{\mathfrak{s o}_{2 n}, k}$ do not depend on the rank $n$.

Proof By Theorem 4.1.1 we have

$$
\operatorname{char}\left(H^{k}\left(\mathfrak{s o}_{N}\right)\right)=\sum_{\lambda \in \mathcal{P}_{n}} K_{\lambda, \emptyset}^{\mathfrak{s} \mathfrak{o}_{N}, k} s_{\lambda}^{\mathfrak{s o}_{N}} \text { and } \operatorname{char}\left(H^{k}\left(\mathfrak{s p}_{2 n}\right)\right)=\sum_{\lambda \in \mathcal{P}_{n}} K_{\lambda, \emptyset}^{\mathfrak{s p} \mathfrak{p}_{2 n}, k} s_{\lambda}^{\mathfrak{s p}_{2 n}}
$$

The Proposition then follows by identifying the coefficients appearing in these decompositions with those appearing in (31).

Thus the coefficients $K_{\lambda, \emptyset}^{\mathfrak{g}, k}$ depend only on $\lambda, k$ and the type $X=C$ or $X \in\{B, D\}$ of the Lie algebra considered when $\operatorname{rank}(\mathfrak{g}) \geq 2 k$. Proposition 4.1 .2 can also be rewritten in the form

$$
\lim _{n \rightarrow \infty} K_{\lambda, \emptyset}^{\mathfrak{s p} p_{2 n}, k}=K_{\lambda, \emptyset}^{\mathfrak{s p}, k} \quad \text { and } \quad \lim _{N \rightarrow \infty} K_{\lambda, \emptyset}^{\mathfrak{s o} \mathfrak{o}_{N}, k}=K_{\lambda, \emptyset}^{\mathfrak{s o}, k}
$$

\subsection{Recurrence formulas for the Lusztig $q$-analogues}

We now recall recurrence formulas established in [12] and [13] which permit to express the Lusztig $q$-analogues associated to the root system of type $X_{n} \in$ $\left\{B_{n}, C_{n}, D_{n}\right\}$ in terms of those associated to the root system of type $X_{n-1}$. They can be regarded as generalizations of the Morris recurrence formula which holds for the Lusztig $q$-analogues of type $A$. 
Suppose $\mathfrak{g}$ is of rank $n$ and consider $v \in \mathcal{P}_{m}$ with $m<n$. For any nonnegative integer $l$, the decomposition of the $\mathfrak{g}$-module $V^{\mathfrak{g}}(\gamma) \otimes V^{\mathfrak{g}}(l)$ into its irreducible components can be written

$$
V^{\mathfrak{g}}(\gamma) \otimes V^{\mathfrak{g}}(l) \simeq \bigoplus_{\lambda \in \mathcal{P}_{n}} V^{\mathfrak{g}}(\lambda)^{\oplus p_{\gamma, l}^{\mathfrak{g}, \lambda}}
$$

This decomposition can be regarded as the analogue of the Pieri rule in type $X_{n}$.

Remark The multiplicities $p_{\gamma, l}^{\mathfrak{g}, \lambda}$ are not necessarily equal to 0 or 1 as in the original Pieri rule (i.e. for $\mathfrak{g l}_{n}$ ). Moreover we can have $p_{\gamma, l}^{\mathfrak{g}, \lambda} \neq 0$ when $|\lambda|<|\gamma|+l$. Nevertheless, when $|\lambda|=|\gamma|+l$, one shows that the Pieri rule for type $X_{n}$ coincide with the original one. It means that $p_{\gamma, l}^{\mathfrak{g}, \lambda}=1$ if $\lambda$ is obtained by adding an horizontal strip of length $l$ to $\gamma$ (i.e. the $l$ boxes of $\lambda / \gamma$ belong to distinct columns) and $p_{\gamma, l}^{\mathfrak{g}, \lambda}=0$ otherwise.

The following Lemma is a consequence of Proposition 2.1.2.

Lemma 4.2.1 Consider $\gamma \in \mathcal{P}_{m}$ and $l \in \mathbb{N}$. Suppose that $\mathfrak{g}$ is a Lie algebra of type $X_{n} \in\left\{B_{n}, C_{n}, D_{n}\right\}$ with $n>m$. Then the multiplicities $p_{\gamma, l}^{\mathfrak{g}, \lambda}$ are independent of the rank $n$ and the type $X \in\{B, C, D\}$ of $\mathfrak{g}$.

We set

$$
p_{\gamma, l}^{\lambda}=\lim _{n \rightarrow \infty} p_{\gamma, l}^{\mathfrak{g}, \lambda}
$$

Let $\mu, v \in \mathcal{P}_{m}$. We can suppose $v \geq \mu$ for the dominance order, since otherwise $K_{\nu, \mu}^{\mathfrak{g}}(q)=0$. Write $p$ maximal in $\{1, \ldots, m\}$ such that $v_{p}-p-\mu_{1}+1 \geq 0$. For any $s \in\{1, \ldots, p\}$ let $\gamma(s)$ be the partition of length $m-1$ such that

$$
\gamma(s)= \begin{cases}\left(v_{2}, \ldots, v_{m}\right) & \text { if } s=1 \\ \left(v_{1}+1, v_{2}+1, \ldots, v_{s-1}+1, v_{s+1}, \ldots, v_{m}\right) & \text { if } s \geq 2\end{cases}
$$

Finally set

$$
R_{s}=v_{s}-s-\mu_{1}+1
$$

\section{Theorem 4.2.2 [13]}

With the above notation, we have for any partitions $\lambda, \mu \in \mathcal{P}_{m}$ :

(i): $K_{\nu, \mu}^{\mathfrak{s o}_{2 n+1}}(q)=\sum_{s=1}^{p}(-1)^{s-1} \times q^{R_{s}} \times \sum_{r+2 a=R_{s}} \sum_{\lambda \in \mathcal{P}_{m-1}} p_{\gamma(s), r}^{\mathfrak{s o}_{2 n-1}, \lambda} K_{\lambda, \mu^{b}}^{\mathfrak{s o}_{2 n-1}}(q)$

(ii): $\quad K_{v, \mu}^{\mathfrak{s p}_{2 n}}(q)=\sum_{s=1}^{p}(-1)^{s-1} \times \sum_{r+2 a=R_{s}} q^{r+a} \sum_{\lambda \in \mathcal{P}_{m-1}} p_{\gamma(s), r}^{\mathfrak{s p}_{2 n-2}, \lambda} K_{\lambda, \mu^{b}}^{\mathfrak{s p}_{2 n-2}}(q)$

(iii): $\quad K_{\nu, \mu}^{\mathfrak{s o}_{2 n}}(q)=\sum_{s=1}^{p}(-1)^{s-1} \times q^{R_{s}} \times \sum_{r+2 a=R_{s}} \sum_{\lambda \in \mathcal{P}_{m-1}} p_{\gamma(s), r}^{\mathfrak{s o}_{2 n-2, \lambda}} K_{\lambda, \mu^{b}}^{\mathfrak{s o}_{2 n-2}}(q)$

where $a \in \mathbb{N}$ and $\mu^{b}=\left(\mu_{2}, \ldots, \mu_{m}\right)$. 
Since the integer $p$ and the partitions $\gamma(s)$ defined above do not depend on the rank of $\mathfrak{g}$, we obtain from Lemma 4.2.1 and Theorem 4.2.2:

\section{Corollary 4.2.3 (of Theorem 4.2.2)}

For any partitions $\lambda, \mu \in \mathcal{P}_{m}$ and any integer $n \geq m$

(i): $\quad K_{v, \mu}^{\mathfrak{s o} \mathfrak{o}_{2 n+1}}(q)=\sum_{s=1}^{p}(-1)^{s-1} \times q^{R_{s}} \times \sum_{r+2 a=R_{s}} \sum_{\lambda \in \mathcal{P}_{m-1}} p_{\gamma(s), r}^{\lambda} K_{\lambda, \mu^{b}}^{\mathfrak{s o}_{2 n-1}}(q)$

(ii): $\quad K_{v, \mu}^{\mathfrak{s p} p_{2 n}}(q)=\sum_{s=1}^{p}(-1)^{s-1} \times \sum_{r+2 a=R_{s}} q^{r+a} \sum_{\lambda \in \mathcal{P}_{m-1}} p_{\gamma(s), r}^{\lambda} K_{\lambda, \mu^{b}}^{\mathfrak{s p}_{2 n-2}}(q)$

(iii): $\quad K_{\nu, \mu}^{\mathfrak{s o}_{2 n}}(q)=\sum_{s=1}^{p}(-1)^{s-1} \times q^{R_{s}} \times \sum_{r+2 a=R_{s}} \sum_{\lambda \in \mathcal{P}_{m-1}} p_{\gamma(s), r}^{\lambda} K_{\lambda, \mu^{b}}^{\mathfrak{s o}_{2 n-2}}(q)$

where $a \in \mathbb{N}$ and $\mu^{b}=\left(\mu_{2}, \ldots, \mu_{m}\right)$.

By using similar arguments to those given in Example 4 page 243 of [15], one shows that the polynomials $K_{\lambda, \mu}^{\mathfrak{g}}(q)$ are monic of degree

$$
\begin{cases}\sum_{i=1}^{n}(n-i+1)\left(\lambda_{i}-\mu_{i}\right) & \text { for } \mathfrak{g}=\mathfrak{s o}_{2 n+1}, \\ \sum_{i=1}^{n}(n-i+1 / 2)\left(\lambda_{i}-\mu_{i}\right) & \text { for } \mathfrak{g}=\mathfrak{s p}_{2 n}, \\ \sum_{i=1}^{n}(n-i)\left(\lambda_{i}-\mu_{i}\right) & \text { for } \mathfrak{g}=\mathfrak{s o}_{2 n} .\end{cases}
$$

Thanks to the recurrence formulas of Theorem 4.2.2, one can derive a lower bound for the lowest degree appearing in $K_{\nu, \mu}^{\mathfrak{g}}(q)$ when $K_{v, \mu}^{\mathfrak{g}}(q) \neq 0$.

Proposition 4.2.4 For any partitions $v, \mu \in \mathcal{P}_{n}$, either $K_{v}^{\mathfrak{g}}, \mu(q)$ is zero or its lowest degree of $q$ is at least $\frac{|v|-|\mu|}{2}$.

Proof We give the proof for $\mathfrak{g}=\mathfrak{s p}_{2 n}$, the arguments are essentially the same for $\mathfrak{g}=\mathfrak{s o}_{2 n+1}$ and $\mathfrak{g}=\mathfrak{s o}_{2 n}$. We proceed by induction on $n$. For $n=1$, one has $K_{v, \mu}^{\mathfrak{s p}_{2}}(q)=0$ or $K_{v, \mu}^{\mathfrak{s p}}(q)=q \frac{|\lambda|-|\mu|}{2}$. Now suppose the lowest degree of $q$ in $K_{\lambda, \mu^{b}}^{\mathfrak{s p}_{2 n-2}}(q)$ is at least $\frac{|\lambda|-\left|\mu^{\mathrm{b}}\right|}{2}$ or $K_{\lambda, \mu^{\mathrm{b}}}^{\mathfrak{s p}_{2 n-2}}(q)=0$. Consider $v, \mu \in \mathcal{P}_{n}$ such that $K_{v, \mu}^{\mathfrak{s p}_{2 n}}(q) \neq 0$. We apply recurrence formula (ii) of Theorem 4.2.2. Since $K_{v, \mu}^{\mathfrak{s} \mathfrak{p}_{2 n}}(q) \neq$ 0 , there exist integers $s$ and $r \leq R_{s}$ such that $K_{\lambda, \mu^{b}}^{\mathfrak{s p}_{2 n-2}}(q) \neq 0$ with $p_{\gamma(s), r}^{\mathfrak{s p}_{2 n-2}, \lambda} \neq 0$. One then have $|\lambda| \geq|\gamma(s)|-r$. The definition (32) of $\gamma(s)$ gives $|\gamma(s)|=|\nu|-$ $v_{s}+s-1$. By the induction hypothesis, the lowest degree of $q$ in the polynomial $q^{r+a} K_{\lambda, \mu^{b}}^{\mathfrak{s p}}(q)$ is at least

$$
d=r+a+\frac{|\lambda|-|\mu|+\mu_{1}}{2} \geq \frac{1}{2} r+a+\frac{|\nu|-v_{s}+s-1-|\mu|+\mu_{1}}{2} .
$$


On the other hand, we have $\frac{1}{2} r+a=\frac{1}{2} R_{s}$ for $r+2 a=R_{s}$. Recall that $R_{s}=v_{s}-$ $s-\mu_{1}+1$. This finally gives

$$
d \geq \frac{v_{s}-s-\mu_{1}+1}{2}+\frac{|v|-v_{s}+s-1-|\mu|+\mu_{1}}{2} \geq \frac{|\nu|-|\mu|}{2}
$$

Remark By the previous proposition, the coefficients $K_{v, \mu}^{\mathfrak{g}, k}$ are all equal to zero when $k<\frac{|v|-|\mu|}{2}$. This implies in particular that we have the decomposition

$$
Q_{\mu}^{\prime \mathfrak{g}}=\sum_{\nu \in \mathcal{P}_{n}} K_{v, \mu}^{\mathfrak{g}}(q) s_{v}^{\mathfrak{g}}=\sum_{k \geq 0} \sum_{v \in \mathcal{P}_{n}[2 k+|\mu|]} K_{v, \mu}^{\mathfrak{g}, k} s_{v}^{\mathfrak{g}} q^{k}
$$

for the Hall-Littlewood functions $Q_{\mu}^{\prime \mathfrak{g}}$.

The Lemma below will be useful to derive the recurrence formulas of Section 5.2.

Lemma 4.2.5 The partitions $\lambda$ appearing in the right hand side of the previous formulas for which there exists a pair $(\gamma(s), r)$ such that $p_{\gamma(s), r}^{\lambda} \neq 0$ must verify one of the following assertions:

1. $\lambda=v$ and then $\mu=\emptyset, s=1, r=R_{1}=v_{1}, \gamma_{1}=v^{b}$,

2. $|\lambda|<|\nu|$,

3. $|\lambda|=|\nu|$ with $\lambda \neq v$ and then $\mu=\emptyset$ and $\left|\lambda^{b}\right|<\left|v^{b}\right|$.

In particular $|\lambda|=|\nu|$ only if $\mu=\emptyset$.

Proof Consider $\lambda$ and $(\gamma(s), r)$ such that $p_{\gamma(s), r}^{\lambda} \neq 0$. We must have $|\lambda| \leq r+$ $|\gamma(s)|=R_{s}-\left(R_{s}-r\right)+|\gamma(s)|$. By definition of $R_{s}$ (33) and $\gamma(s)$ (32) we obtain $|\lambda| \leq|\nu|-\mu_{1}-\left(R_{s}-r\right)$. Thus $|\lambda|<|\nu|$ when $\mu \neq \emptyset$ and $|\lambda|=|\nu|$ only if $\mu=\emptyset$ and $r=R_{S}$. This permits to restrict ourselves to the case when $\mu=\emptyset,|\lambda|=|\nu|$ and $r=R_{s}$.

Suppose first $\lambda=v$. Then we must have $s=1$. Otherwise $\gamma(s)_{1}=v_{1}+1$ and we would have $\lambda_{1}>v_{1}$. This gives $\mu=\emptyset, s=1, r=R_{1}=v_{1}$ and $\gamma_{1}=v^{b}$ as desired.

Now suppose $|\lambda|=|\nu|$ with $\lambda \neq \nu$. Observe that $\left|\lambda^{b}\right|<\left|\nu^{b}\right|$ if and only if $\lambda_{1}>\nu_{1}$. When $s>1$, we have $\gamma(s)_{1}=v_{1}+1>v_{1}$, thus $\lambda_{1}>v_{1}$ (see Remark before Lemma 4.2.1). When $s=1$, we have $\gamma(1)=\left(v_{2}, \ldots, v_{n}\right)=v^{b}$ and $r=v_{1}$. Since $|\lambda|=|v|$ and $p_{v^{b}, \nu_{1}}^{\lambda} \neq 0, \lambda$ is obtained by adding a horizontal strip of length $v_{1}$ on $v^{b}$. This implies $\lambda_{1}>v_{1}$ because the number of columns in $v^{b}$ is equal to $v_{2} \leq v_{1}$ and we have assumed $\lambda \neq v$.

4.3 Stabilization of the coefficients $K_{\lambda, \mu}^{\mathfrak{g}, k}$

Theorem 4.3.1 Consider $m$ and a nonnegative integers and $v, \mu$ two partitions such that $\nu \in \mathcal{P}_{m}$ and $\mu \in \mathcal{P}_{a}$. Let $\mathfrak{g}$ be a Lie algebra of type $X_{n} \in\left\{B_{n}, C_{n}, D_{n}\right\}$ and $k$ a nonnegative integer. Then for any $n \geq 2 k+a$, the coefficients $K_{\nu, \mu}^{\mathfrak{g}, k}$ do not depend on the rank $n$ of $\mathfrak{g}$. Under these hypothesis, we have $K_{\nu, \mu}^{\mathfrak{s o}_{2 n+1}, k}=K_{\nu, \mu}^{\mathfrak{s o}_{2 n}, k}$. 
Proof Suppose first $\mathfrak{g}=\mathfrak{s o}_{2 n+1}$. We proceed by induction on $a$. Note that we can

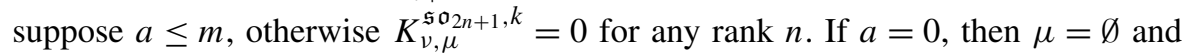
the theorem follows directly from Proposition 4.1.2. Suppose now our theorem true for any partition $\mu^{b}$ of length $a-1$ with $1 \leq a \leq m$ and consider $\mu$ a partition of length $a$. We then apply the recurrence formulas of Corollary 4.2.3. It follows from (33) that the integers $R_{s}$ appearing in these formulas do not depend on the rank $n$ considered. This is also true for the multiplicities $p_{\gamma(s), r}^{\lambda}$.

By our induction hypothesis, for any $p \in \mathbb{N}$, the coefficients $K_{\lambda, \mu^{b}}^{\mathfrak{s o}_{2 n-1}, p}$ are independent of $n$. Indeed $\mu^{b} \in \mathcal{P}_{a-1}$ and $\mathfrak{s o}_{2 n-1}$ has rank $n-1 \geq 2 k+a-1$. The recurrence formulas of Corollary 4.2.3 imply that each coefficient $K_{v, \mu}^{\mathfrak{s o}_{2 n+1}, k}$ can be expressed in terms of the coefficients $K_{\lambda, \mu^{b}}^{\mathfrak{s o}_{2 n-1}, p}$, the integers $R_{S}$ and $p_{\gamma(s), r}^{\lambda}$. Moreover, this decomposition is independent of $n$. Hence $K_{v, \mu}^{\mathfrak{s o}_{2 n+1}, k}$ does not depend on $n$. By using similar arguments, we prove that $K_{v, \mu}^{\mathfrak{g}, k}$ does not depend on $n$ when $\mathfrak{g}=\mathfrak{s p}_{2 n}$ or $\mathfrak{s o}_{2 n}$.

The equality $K_{v, \mu}^{\mathfrak{s o}_{2 n+1}, k}=K_{v, \mu}^{\mathfrak{s o}_{2 n}, k}$ is again obtained by induction on $a$. It is true for $a=0$ by Proposition 4.1.2 and the induction follows from the fact that the recurrence formulas of Corollary 4.2.3 are the same for $\mathfrak{s o}_{2 n+1}$ and $\mathfrak{s o}_{2 n}$.

Remark The arguments used in the previous proof imply that it is possible to decompose any Lusztig $q$-analogue $K_{v, \mu}^{\mathfrak{g}}(q)$ such that $\mu \neq \emptyset$ in terms of the Lusztig $q$-analogues $K_{\lambda, \emptyset}^{\mathfrak{g}}(q)$. Moreover this decomposition is independent of the rank $n$ providing this rank is sufficiently large. In this case the decomposition is the same for $K_{\mathcal{v}, \mu}^{\mathfrak{s o}_{2 n+1}}(q)$ and $K_{\mathcal{v}, \mu}^{\mathfrak{s o}_{2 n}}(q)$. Nevertheless, these two polynomials do not coincide since $K_{v, \emptyset}^{\mathfrak{s o}_{2 n+1}}(q) \neq K_{v, \emptyset}^{\mathfrak{s o} 2 n}(q)$ in general. The previous Theorem also establishes the equality $K_{v, \mu}^{\mathfrak{s o}_{2 n+1, k}}(q)=K_{v, \mu}^{\mathfrak{s o} \mathfrak{s}_{2 n, k}}(q)$ for any $k \leq \frac{n-a}{2}$ where $a$ is the number of nonzero parts in $\mu$.

By Theorem 4.3.1, it makes sense to set

$$
K_{\nu, \mu}^{\mathfrak{s o}, k}=\lim _{n \rightarrow \infty} K_{\mathcal{v}, \mu}^{\mathfrak{s o}_{2 n+1}, k}=\lim _{n \rightarrow \infty} K_{v, \mu}^{\mathfrak{s o}_{2 n}, k} \quad \text { and } \quad K_{\nu, \mu}^{\mathfrak{s p}, k}=\lim _{n \rightarrow \infty} K_{\nu, \mu}^{\mathfrak{s p}_{2 n}, k} .
$$

\subsection{A reformulation in terms of the Brylinski-Kostant filtration}

Recall that the Lusztig $q$-analogue $K_{\lambda, \mu}^{\mathfrak{g}}(q)$ can also be characterized from the Brylinski-Kostant filtration on the weight space $V^{\mathfrak{g}}(\lambda)_{\mu}$ [1]. Take $e=e_{1}+\cdots+e_{n} \in$ $\mathfrak{u}_{+}$for a principal nilpotent in $\mathfrak{g}$ compatible with $\mathfrak{h}$. The $e$-filtration of $V^{\mathfrak{g}}(\lambda)_{\mu}$ is the finite filtration $J_{e}\left(V^{\mathfrak{g}}(\lambda)_{\mu}\right)$ such that

$$
\{0\} \subset J_{e}^{0}\left(V^{\mathfrak{g}}(\lambda)_{\mu}\right) \subset J_{e}^{1}\left(V^{\mathfrak{g}}(\lambda)_{\mu}\right) \subset \cdots
$$

where for any nonnegative integer $k$,

$$
J_{e}^{k}\left(V^{\mathfrak{g}}(\lambda)_{\mu}\right)=\left\{v \in V^{\mathfrak{g}}(\lambda)_{\mu} \mid e^{k+1}(v)=0\right\} .
$$

For completeness we also set $J_{e}^{-1}\left(V^{\mathfrak{g}}(\lambda)_{\mu}\right)=\{0\}$. The following theorem is a consequence of the main result of [1]. 
Theorem 4.4.1 (Brylinski) Consider $m$ a nonnegative integer and $\lambda, \mu \in \mathcal{P}_{m}$. Let $\mathfrak{g}$ be a Lie algebra of type $X_{n} \in\left\{B_{n}, C_{n}, D_{n}\right\}$. Then

$$
K_{\lambda, \mu}^{\mathfrak{g}}(q)=\sum_{k \geq 0} \operatorname{dim}\left(J_{e}^{k}\left(V^{\mathfrak{g}}(\lambda)_{\mu}\right) / J_{e}^{k-1}\left(V^{\mathfrak{g}}(\lambda)_{\mu}\right)\right) q^{k}
$$

By using Theorem 4.3.1, the dimension of the space $J_{e}^{k}\left(V^{\mathfrak{g}}(\lambda)_{\mu}\right)$ does not depend on the rank $n$ of $\mathfrak{g}$ providing $n$ is sufficiently large. More precisely, we have:

Theorem 4.4.2 Consider $\lambda \in \mathcal{P}_{m}, \mu \in \mathcal{P}_{a}$ and $k \in \mathbb{N}$. Let $\mathfrak{g}$ be a Lie algebra of type $X_{n} \in\left\{B_{n}, C_{n} D_{n}\right\}$ with $n \geq 2 k+a$. Then $\operatorname{dim}\left(J_{e}^{k}\left(V^{\mathfrak{g}}(\lambda)_{\mu}\right)\right)$ is independent of the rank $n$ of $\mathfrak{g}$. Moreover we have in this case

$$
\operatorname{dim}\left(J_{e}^{k}\left(V^{\mathfrak{s o} \mathfrak{o}_{2 n+1}}(\lambda)_{\mu}\right)\right)=\operatorname{dim}\left(J_{e}^{k}\left(V^{\mathfrak{s o} \mathfrak{o}_{2 n}}(\lambda)_{\mu}\right)\right) .
$$

Proof We deduce from Theorem 4.3.1 and (36) that the coefficients

$$
K_{\lambda, \mu}^{X, k}=\operatorname{dim}\left(J_{e}^{k}\left(V^{\mathfrak{g}}(\lambda)_{\mu}\right)\right)-\operatorname{dim}\left(J_{e}^{k-1}\left(V^{\mathfrak{g}}(\lambda)_{\mu}\right)\right)
$$

do not depend on $n$ providing $n \geq 2 k+a$. Under this hypothesis, one can write

$$
\operatorname{dim}\left(J_{e}^{k}\left(V^{\mathfrak{s o}_{N}}(\lambda)_{\mu}\right)\right)=\sum_{a=0}^{k} K_{\lambda, \mu}^{\mathfrak{s o}, a} \quad \text { and } \quad \operatorname{dim}\left(J_{e}^{k}\left(V^{\mathfrak{s p} \mathfrak{p}_{2 n}}(\lambda)_{\mu}\right)\right)=\sum_{a=0}^{k} K_{\lambda, \mu}^{\mathfrak{s p}, a} .
$$

Hence $\operatorname{dim}\left(J_{e}^{k}\left(V^{\mathfrak{s o}_{N}}(\lambda)_{\mu}\right)\right)$ and $\operatorname{dim}\left(J_{e}^{k}\left(V^{\mathfrak{s p}_{2 n}}(\lambda)_{\mu}\right)\right)$ are independent of $n$. Moreover, we have

$$
\operatorname{dim}\left(J_{e}^{k}\left(V^{\mathfrak{s o}_{2 n+1}}(\lambda)_{\mu}\right)\right)=\operatorname{dim}\left(J_{e}^{k}\left(V^{\mathfrak{s o} 2 n}(\lambda)_{\mu}\right)\right)
$$

Remark In general, the spaces $J_{e}^{k}\left(V^{\mathfrak{g}}(\lambda)\right)_{\mu}$ depend on the rank $n \geq 2 k+a$ considered although their dimension does not. It seems to be an interesting problem to find explicit bases for the weight spaces $J_{e}^{k}\left(V^{\mathfrak{g}}(\lambda)_{\mu}\right)$.

\section{Limit of Lusztig $q$-analogues}

5.1 The formal series $K_{\lambda, \mu}^{\mathfrak{s o}}(q)$ and $K_{\lambda, \mu}^{\mathfrak{s p}}(q)$

The results of Theorem 4.3.1 suggest to introduce the formal series $K_{\lambda, \mu}^{\mathfrak{s o}}(q)$ and $K_{\lambda, \mu}^{\mathfrak{s p}}(q)$ defined by

$$
K_{\lambda, \mu}^{\mathfrak{s o}}(q)=\sum_{r \geq 0} K_{\lambda, \mu}^{\mathfrak{s o}, k} q^{k} \in \mathbb{N}[[q]] \quad \text { and } \quad K_{\lambda, \mu}^{\mathfrak{s p}}(q)=\sum_{r \geq 0} K_{\lambda, \mu}^{\mathfrak{s p}, k} q^{k} \in \mathbb{N}[[q]]
$$

where the coefficients $K_{\lambda, \mu}^{\mathfrak{s o}, k}$ and $K_{\lambda, \mu}^{\mathfrak{s p}, k}$ are those defined in (35). Then, $K_{\lambda, \mu}^{\mathfrak{s o}}(q)$ and $K_{\lambda, \mu}^{\mathfrak{s p}}(q)$ can be regarded as the limits of the Lusztig $q$-analogues $K_{\lambda, \mu}^{\mathfrak{s o}_{N}}(q)$ and $K_{\lambda, \mu}^{\mathfrak{s p}}(q)$ when the rank $n$ of $\mathfrak{g}$ tends to infinity. 
Moreover, we have

$$
\operatorname{char}_{q}(\mathrm{H}(\mathfrak{s p}))=\sum_{\lambda \in \mathcal{P}} K_{\lambda, \emptyset}^{\mathfrak{s p}}(q) \mathrm{s}_{\lambda}^{\mathfrak{s p}} \quad \text { and } \quad \operatorname{char}_{q}(\mathrm{H}(\mathfrak{s o}))=\sum_{\lambda \in \mathcal{P}} K_{\lambda, \emptyset}^{\mathfrak{s o}}(q) \mathrm{s}_{\lambda}^{\mathfrak{s o}}
$$

\section{Remark}

(i) When $\mathfrak{g}=\mathfrak{s p}_{2 n}$ or $\mathfrak{s o}_{2 n}, K_{\lambda, \mu}^{\mathfrak{g}}(q)=0$ for any partitions $\lambda, \mu$ such that $|\lambda|-|\mu|$ is odd. Thus for such partitions we have also $K_{\lambda, \mu}^{\mathfrak{s p}}(q)=K_{\lambda, \mu}^{\mathfrak{s o}}(q)=0$.

(ii) Observe that we may have $K_{\lambda, \mu}^{\mathfrak{s o}_{2 n+1}}(q) \neq 0$ even if $|\lambda|-|\mu|$ is odd. In this case we have

$$
\lim _{n \rightarrow \infty} K_{\lambda, \mu}^{\mathfrak{s o}_{2 n+1, k}}(q)=0
$$

for any nonnegative integer $k$. Take as an example $\lambda=(1)$ and $\mu=\emptyset$. Then $K_{(1), \emptyset}^{\mathfrak{s o} 2 n+1}(q)=q^{n-1}$ for any rank $n \geq 2$. Thus (38) is verified for any fixed degree $k$.

\subsection{Recurrence formulas for the series $K_{\lambda, \mu}^{\mathfrak{s o}}(q)$ and $K_{\lambda, \mu}^{\mathfrak{s p}}(q)$}

By taking the limit when $n$ tends to infinity in the formulas of Corollary 4.2.3 (which do not depend on $n$ ), we obtain the identities

$$
\begin{aligned}
& K_{\nu, \mu}^{\mathfrak{s o}}(q)=\sum_{s=1}^{p}(-1)^{s-1} \times q^{R_{s}} \times \sum_{r+2 a=R_{s}} \sum_{\lambda \in \mathcal{P}} p_{\gamma(s), r}^{\lambda} K_{\lambda, \mu^{\mathfrak{b}}}^{\mathfrak{s o}}(q), \\
& K_{\nu, \mu}^{\mathfrak{s p}}(q)=\sum_{s=1}^{p}(-1)^{s-1} \times \sum_{r+2 a=R_{s}} q^{r+a} \sum_{\lambda \in \mathcal{P}} p_{\gamma(s), r}^{\lambda} K_{\lambda, \mu^{b}}^{\mathfrak{s p}}(q)
\end{aligned}
$$

where $a \in \mathbb{N}$ and $\mu^{b}=\left(\mu_{2}, \ldots, \mu_{m}\right)$. These identities yield recurrence formulas for the limit of $q$-analogues.

To see it, suppose first $\mu \neq \emptyset$. By Lemma 4.2.5, the formal series $K_{\lambda, \mu^{b}}^{\mathfrak{s o}}(q)$ and $K_{\lambda, \mu^{b}}^{\mathfrak{s p}}(q)$ appearing in the right hand sides of (39) are such that $|\lambda|<|\nu|$. Thus formulas (39) permit to express the series $K_{v, \mu}^{\mathfrak{s o}}(q)$ and $K_{\nu, \mu}^{\mathfrak{s p}}(q)$ respectively in terms of the series $K_{\lambda, \mu^{b}}^{\mathfrak{s o}}(q)$ and $K_{\lambda, \mu^{b}}^{\mathfrak{s p}}(q)$ with $|\lambda|<|\nu|$.

Now suppose $\mu=\emptyset$. Then by Lemma 4.2.5, $K_{\lambda, \emptyset}^{\mathfrak{s o}}(q)$ and $K_{\lambda, \emptyset}^{\mathfrak{s p}}(q)$ also appear in the right hand sides of (39) when $\gamma(s)=\gamma(1)=v^{b}, R_{s}=R_{1}=v_{1}$. We can write

$$
\begin{aligned}
K_{\nu, \emptyset}^{\mathfrak{s o}}(q)= & \frac{1}{1-q^{\nu_{1}}}\left(q^{\nu_{1}} \sum_{\substack{r+2 a=\nu_{1} \\
a \neq 0}} \sum_{\lambda \in \mathcal{P}} p_{\nu^{b}, r}^{\lambda} K_{\lambda, \emptyset}^{\mathfrak{s o}}(q)\right. \\
& \left.+\sum_{s=2}^{p-1}(-1)^{s-1} \times q^{R_{s}} \sum_{r+2 a=R_{s}} \sum_{\lambda \in \mathcal{P}} p_{\gamma(s), r}^{\lambda} K_{\lambda, \emptyset}^{\mathfrak{s o}(q)}\right),
\end{aligned}
$$




$$
\begin{aligned}
K_{\nu, \emptyset}^{\mathfrak{s p}}(q)= & \frac{1}{1-q^{\nu_{1}}}\left(\sum_{\substack{r+2 a=\nu_{1} \\
a \neq 0}} q^{r+a} \sum_{\lambda \in \mathcal{P}} p_{\nu^{b}, r}^{\lambda} K_{\lambda, \emptyset}^{\mathfrak{s p}}(q)\right. \\
& \left.+\sum_{s=2}^{p}(-1)^{s-1} \times \sum_{r+2 a=R_{s}} q^{r+a} \sum_{\lambda \in \mathcal{P}} p_{\gamma(s), r}^{\lambda} K_{\lambda, \emptyset}^{\mathfrak{s p}}(q)\right)
\end{aligned}
$$

where the series $K_{\lambda, \emptyset}^{\mathfrak{s o}}(q)$ and $K_{\lambda, \emptyset}^{\mathfrak{s p}}(q)$ appearing in the right hand sides are such that $|\lambda|<|\nu|$ or, $|\lambda|=|\nu|$ and $\left|\lambda^{b}\right|<\left|\mu^{b}\right|$. Thus formulas (40) permit to express the series $K_{\nu, \emptyset}^{\mathfrak{s o}}(q)$ and $K_{\nu, \emptyset}^{\mathfrak{s p}}(q)$ respectively in terms of the series $K_{\lambda, \emptyset}^{\mathfrak{s o}}(q)$ and $K_{\lambda, \emptyset}^{\mathfrak{s p}}(q)$ with $|\lambda|<|\nu|$ or, $|\lambda|=|\nu|$ and $\left|\lambda^{b}\right|<\left|\mu^{b}\right|$. Observe that $|\lambda|+\left|\lambda^{b}\right|<|\nu|+\left|\nu^{b}\right|$. Hence one can compute the series $K_{v, \emptyset}^{\mathfrak{s o}}(q)$ and $K_{\nu, \emptyset}^{\mathfrak{s p}}(q)$ by induction on $|v|+\left|v^{b}\right|$ starting from the obvious identity $K_{\emptyset, \emptyset}^{\mathfrak{s o}}(q)=K_{\emptyset, \emptyset}^{\mathfrak{s p}}(q)=1$.

Finally the series $K_{v, \mu}^{\mathfrak{s o}}(q)$ and $K_{v, \mu}^{\mathfrak{s p}}(q)$ with $\mu \neq \emptyset$ can be computed by induction on $|v|$ from the series $K_{v, \emptyset}^{\mathfrak{s o}}(q)$ and $K_{v, \emptyset}^{\mathfrak{s p}}(q)$. We give in Proposition 5.4.1 explicit formulas for $K_{v, \emptyset}^{\mathfrak{s o}}(q)$ and $K_{v, \emptyset}^{\mathfrak{s p}}(q)$ when $v$ is a row or a column partition.

5.3 A duality between the series $K_{\lambda, \emptyset}^{\mathfrak{s o}}(q)$ and $K_{\lambda^{\prime}, \emptyset}^{\mathfrak{s p}}(q)$.

Proposition 5.3.1 For any partition $\lambda$ we have the duality

$$
K_{\lambda, \emptyset}^{\mathfrak{s o}}(q)=K_{\lambda^{\prime}, \emptyset}^{\mathfrak{s p}}(q)
$$

between the limits of the orthogonal and symplectic Lusztig q-analogues corresponding to the weight 0.

Proof We have

$$
\operatorname{char}_{q}(\mathrm{H}(\mathfrak{s o}))=\prod_{i \geq 1}\left(1-q^{2 i}\right) \operatorname{char}_{q}(\mathrm{~S}(\mathfrak{s o}))
$$

and

$$
\operatorname{char}_{q}(\mathrm{H}(\mathfrak{s p}))=\prod_{i \geq 1}\left(1-q^{2 i}\right) \operatorname{char}_{q}(\mathrm{~S}(\mathfrak{s p})) .
$$

Moreover by Corollary 3.2.4, $\varphi\left(\operatorname{char}\left(S^{k}(\mathfrak{s o})\right)\right)=\operatorname{char}\left(S^{k}(\mathfrak{s p})\right)$ for any nonnegative integer $k$. This implies the equality

$$
\varphi\left(\operatorname{char}\left(\mathrm{H}^{k}(\mathfrak{s o})\right)\right)=\operatorname{char}\left(\mathrm{H}^{k}(\mathfrak{s p})\right) \quad \text { for any } k \in \mathbb{N} .
$$

Recall that

$$
\operatorname{char}\left(\mathrm{H}^{k}(\mathfrak{s o})\right)=\sum_{\lambda \in \mathcal{P}} K_{\lambda, \emptyset}^{\mathfrak{s o}, k} \mathrm{~S}_{\lambda}^{\mathfrak{s o}} \quad \text { and } \quad \operatorname{char}\left(\mathrm{H}^{k}(\mathfrak{s p})\right)=\sum_{\lambda \in \mathcal{P}} K_{\lambda, \emptyset}^{\mathfrak{s p}, k} \mathrm{~S}_{\lambda}^{\mathfrak{s p}}
$$

By using (41), this gives

$$
\operatorname{char}\left(\mathrm{H}^{k}(\mathfrak{s p})\right)=\sum_{\lambda \in \mathcal{P}} K_{\lambda, \emptyset}^{\mathfrak{s p}, k} \mathrm{~s}_{\lambda}^{\mathfrak{s p}}=\sum_{\lambda \in \mathcal{P}} K_{\lambda, \emptyset}^{\mathfrak{s o}, k} \mathbf{s}_{\lambda^{\prime}}^{\mathfrak{s p}}
$$


Since the map

$$
\iota:\left\{\begin{array}{l}
\mathcal{P} \rightarrow \mathcal{P} \\
\lambda \longmapsto \lambda^{\prime}
\end{array}\right.
$$

is bijective, we must have $K_{\lambda, \emptyset}^{\mathfrak{s p}, k}(q)=K_{\lambda^{\prime}, \emptyset}^{\mathfrak{s o}, k}(q)$ for any nonnegative integer $k$ which proves the proposition.

Remark The duality of the previous theorem does not hold for the Lusztig $q$ analogues, that is $K_{\lambda, \emptyset}^{\mathfrak{s p}_{2 n}}(q) \neq K_{\lambda^{\prime}, \emptyset}^{\mathfrak{s o} \mathfrak{D}_{2 n+1}}(q)$ in general. Nevertheless we have $K_{\lambda, \emptyset}^{\mathfrak{s p}_{2 n}, k}(q)=K_{\lambda^{\prime}, \emptyset}^{\mathfrak{s o}_{N}, k}(q)$ when $k \leq \frac{n}{2}$ according to Proposition 4.1.2.

\subsection{Some explicit formulas}

We give below some explicit formulas for the series $K_{v, \mu}^{\mathfrak{s o}}(q)$ and $K_{v, \mu}^{\mathfrak{s p}}(q)$ when $v$ is a column or a row partition. Note that we have not found such simple formulas for the Lusztig $q$-analogues $K_{\nu, \mu}^{\mathfrak{g}}(q)$ even in the case when $v$ is a row or a column.

Proposition 5.4.1 Consider l a nonnegative integer. Recall that $(2 l)$ and $\left(1^{2 l}\right)$ are the row and column partitions of length and height $2 l$, respectively. We have

$$
\begin{aligned}
& K_{(2 l), \emptyset}^{\mathfrak{s p}}(q)=K_{\left(1^{2 l}\right), \emptyset}^{\mathfrak{s o}}(q)=\frac{q^{l}}{\prod_{i=1}^{l}\left(1-q^{2 i}\right)}, \\
& K_{(2 l), \emptyset}^{\mathfrak{s o}}(q)=K_{\left(1^{2 l}\right), \emptyset}^{\mathfrak{s p}}(q)=\frac{q^{2 l}}{\prod_{i=1}^{l}\left(1-q^{2 i}\right)} .
\end{aligned}
$$

Proof We only give the proof for the first equality of the proposition. The proof for the second one is similar.

We use the recurrence formula (40). We have then $p=1, R_{1}=2 l$ and $\gamma(1)=\emptyset$. Thus $p_{\gamma(1), r}^{\lambda} \neq 0$ only when $\lambda=(r)$ and in this case $p_{\gamma(1), r}^{r}=1$. This yields for any $l \geq 1$

$$
K_{(2 l), \emptyset}^{\mathfrak{s p}}(q)=\frac{1}{1-q^{2 l}} \sum_{\substack{r+2 a=2 l \\ r \neq 2 l}} q^{r+a} K_{(r), \emptyset}^{\mathfrak{s p}}(q)=\frac{q^{l}}{1-q^{2 l}} \sum_{b=0}^{l-1} q^{b} K_{(2 b), \emptyset}^{\mathfrak{s p}}(q)
$$

where the last equality is obtained by setting $r=2 b$. By an immediate induction starting from $K_{\emptyset, \emptyset}^{\mathfrak{s p}}(q)=1$, one derives the desired formula

$$
K_{(2 l), \emptyset}^{\mathfrak{s p}}(q)=\frac{q^{l}}{\prod_{i=1}^{l}\left(1-q^{2 i}\right)}
$$

by using the identity

$$
\sum_{b=0}^{l-1} q^{b} K_{(2 b), \emptyset}^{\mathfrak{s p}}(q)=\sum_{b=0}^{l-1} \frac{q^{2 b}}{\prod_{i=1}^{b}\left(1-q^{2 i}\right)}=\frac{1}{\prod_{i=1}^{l-1}\left(1-q^{2 i}\right)} .
$$

We deduce then $K_{(2 l), \emptyset}^{\mathfrak{s p}}(q)=K_{\left(1^{2 l}\right), \emptyset}^{\mathfrak{s o}}(q)$ from Theorem 5.3.1. 
Corollary 5.4.2 Consider $m$ a nonnegative integer and $\mu$ a partition with $d$ nonzero parts. Then

1. $K_{(m), \mu}^{\mathfrak{s p}}(q) \neq 0$ and $K_{(m), \mu}^{\mathfrak{s o}}(q) \neq 0$ only if $m-|\mu| \in 2 \mathbb{N}$. In this case

$$
K_{(m), \mu}^{\mathfrak{s p}}(q)=q^{h(\mu)} K_{(2 l), \emptyset}^{\mathfrak{s p}}(q)=\frac{q^{h(\mu)+l}}{\prod_{i=1}^{l}\left(1-q^{2 i}\right)}
$$

and

$$
K_{(m), \mu}^{\mathfrak{s o}}(q)=q^{h(\mu)} K_{(2 l), \emptyset}^{\mathfrak{s o}}(q)=\frac{q^{h(\mu)+2 l}}{\prod_{i=1}^{l}\left(1-q^{2 i}\right)}
$$

where $h(\mu)=\sum_{1 \leq i \leq d}(i-1) \mu_{i}$ and $l=\frac{m-|\mu|}{2}$.

2. $K_{\left(1^{m}\right), \mu}^{\mathfrak{s p}}(q) \neq 0$ and $K_{(m), \mu}^{\mathfrak{s o}}(q) \neq 0$ only if $\mu=\left(1^{p}\right)$ with $m-p \in 2 \mathbb{N}$ and in this case

$$
K_{\left(1^{m}\right),\left(1^{p}\right)}^{\mathfrak{s p}}(q)=K_{\left(1^{2 l}\right), \emptyset}^{\mathfrak{s p}}(q)=\frac{q^{2 l}}{\prod_{i=1}^{l}\left(1-q^{2 i}\right)}
$$

and

$$
K_{\left(1^{m}\right),\left(1^{p}\right)}^{\mathfrak{s o}}(q)=K_{\left(1^{2 l}\right), \emptyset}^{\mathfrak{s o}}(q)=\frac{q^{l}}{\prod_{i=1}^{l}\left(1-q^{2 i}\right)}
$$

where $l=\frac{m-|p|}{2}$.

Proof 1. We proceed by induction on the number $d$ of nonzero parts of $\mu$. If $d=0$, the result follows from Proposition 5.4.1. Suppose $d>0$ and apply the recurrence formula (39). We have $p=1, R_{1}=m-\mu_{1}$ and $\gamma(1)=\emptyset$. This gives

$$
K_{(m), \mu}^{\mathfrak{s p}}(q)=\sum_{r+2 a=m-\mu_{1}} q^{r+a} K_{(r), \mu^{b}}^{\mathfrak{s p}}(q)
$$

Since $K_{(r), \mu^{b}}^{\mathfrak{s p}}(q)=0$ when $r<\left|\mu^{b}\right|$, we can suppose $r \geq\left|\mu^{b}\right|$ in the above sum. This gives by using the induction hypothesis

$$
K_{(m), \mu}^{\mathfrak{s p}}(q)=q^{h\left(\mu^{b}\right)} \sum_{r+2 a=m-\mu_{1}} q^{r+a} K_{r-\left|\mu^{b}\right|, \emptyset}^{\mathfrak{s p}}(q) .
$$

We must have $r-\left|\mu^{b}\right| \in 2 \mathbb{N}$, thus we can set $b=\frac{r-\left|\mu^{b}\right|}{2}$. One then obtains

$$
K_{(m), \mu}^{\mathfrak{s p}}(q)=q^{h\left(\mu^{\mathrm{b}}\right)} \sum_{b=0}^{l} q^{\frac{|\mu|+m}{2}+b-\mu_{1}} K_{(2 b), \emptyset}^{\mathfrak{s p}}(q)=q^{h(\mu)} \sum_{b=0}^{l} q^{l+b} K_{(2 b), \emptyset}^{\mathfrak{s p}}(q)
$$


where the last equality follows from the identities $l=\frac{m-|\mu|}{2}$ and $h(\mu)=h\left(\mu^{b}\right)+$ $|\mu|-\mu_{1}$. By using (44), this gives

$$
K_{(m), \mu}^{\mathfrak{s p}}(q)=q^{h(\mu)} \times \frac{q^{l}}{\prod_{i=1}^{l}\left(1-q^{2 i}\right)}=q^{h(\mu)} K_{(2 l), \emptyset}^{\mathfrak{s p}}(q) .
$$

The proof is similar for $K_{(m), \mu}^{\mathfrak{s o}}(q)$.

2. By applying (39), we obtain this time $p=1, R_{1}=0$ and $\gamma(1)=\emptyset$. Hence

$$
K_{\left(1^{m}\right),\left(1^{p}\right)}^{\mathfrak{s p}}(q)=K_{\left(1^{m-1}\right),\left(1^{p-1}\right)}^{\mathfrak{s p}}(q) .
$$

By an immediate induction, this gives $K_{\left(1^{m}\right),\left(1^{p}\right)}^{\mathfrak{s p}}(q)=K_{\left(1^{m-p}\right), \emptyset}^{\mathfrak{s p}}(q)=K_{\left(1^{2 l}\right), \emptyset}^{\mathfrak{s p}}(q)$ and our formula follows from Proposition 5.4.1.

Remark Recurrence formulas (39) and (40) also permit to derive explicit formulas when $v$ is not a row partition. For example we have

$$
\begin{aligned}
& K_{(3,1), \emptyset}^{\mathfrak{s p}}(q)=\frac{q^{3}\left(1+q+q^{2}\right)}{\left(1-q^{2}\right)\left(1-q^{4}\right)}, \quad K_{(2,2), \emptyset}^{\mathfrak{s p}}(q)=\frac{q^{2}}{\left(1-q^{2}\right)^{2}}, \\
& K_{(2,1,1), \emptyset}^{\mathfrak{s p}}(q)=\frac{q^{3}\left(1+q^{2}+q^{3}\right)}{\left(1-q^{2}\right)\left(1-q^{4}\right)} .
\end{aligned}
$$

\section{References}

1. Brylinski, R.-K.: Limits of weight spaces, Lusztig's $q$-analogs and fiberings of adjoint orbits. J. Am. Math. Soc. 29(3), 517-533 (1989)

2. Gupta, R.K.: Generalized exponents via Hall-Littlewood symmetric functions. Bull. Am. Math. Soc. 16(2), 287-291 (1987)

3. Hanlon, P.: On the decomposition of the tensor algebra of the classical Lie algebras. Adv. Math. 56, 238-282 (1985)

4. Goodman, G., Wallach, N.R.: Representation Theory and Invariants of the Classical Groups. Cambridge University Press, Cambridge (2003)

5. Hesselink, W.-H.: Characters of the nullcone. Math. Ann. 252, 179-182 (1980)

6. Kashiwara, M., Nakashima, T.: Crystal graphs for representations of the $q$-analogue of classical Lie algebras. J. Algebra 165, 295-345 (1994)

7. Kato, S.: Spherical functions and a $q$-analogue of Kostant's weight multiplicity formula. Inv. Math. 66, 461-468 (1982)

8. Koike, K., Terada, I.: Young diagrammatic methods for the representation theory of the classical groups of type $B_{n}, C_{n}$ and $D_{n}$. J. Algebra 107, 466-511 (1987)

9. Koike, K., Terada, I.: Young diagrammatic methods for the restriction of representations of complex classical Lie groups to reductive subgroups of maximal rank. Adv. Math. 79, 104-135 (1990)

10. Littlewood, D.-E.: The Theory of Group Characters and Matrix Representations of Groups, 2nd edn. Oxford University Press, Oxford (1958)

11. Lascoux, A., Schützenberger, M.-P.: Sur une conjecture de H.O. Foulkes. CR Acad. Sci. Paris 288, 95-98 (1979)

12. Lecouvey, C.: Kostka-Foulkes polynomials cyclage graphs and charge statistic for the root system $C_{n}$. J. Algebr. Comb. 21, 203-240 (2005)

13. Lecouvey, C.: Combinatorics of crystal graphs and Kostka-Foulkes polynomials for the root systems $B_{n}, C_{n}$ and $D_{n}$. Eur. J. Comb. 27, 526-557 (2006)

14. Lusztig, G.: Singularities, character formulas, and a $q$-analog of weight multiplicities. Astérisque 101-102, 208-227 (1983)

15. Macdonald, I.-G.: Symmetric Functions and Hall Polynomials, 2nd edn. Oxford Mathematical Monograph. Oxford University Press, New York (1995) 